\title{
Spatial chromatic tessellation: conception, interpretation, and implication
}

\author{
Weining Zhu and Qian Yu* \\ Department of Geosciences, University of Massachusetts, Amherst, MA, USA
}

(Received 24 June 2010; final version received 3 November 2010)

\begin{abstract}
A novel spatial tessellation scheme, spatial chromatic tessellation (SCT), is proposed for representing and exploring spaceentity relationship. The objective of this article is briefly introducing the basic concepts, structure, and properties of SCT. Our study mainly focuses on the arranged chromatic diagram (ACD), which is the most typical type of SCT. A few examples show that ACD can be used to analyze spatial topology, statistics, computations, database, and Voronoi diagrams. ACD also contains many implications on spatial analysis theory. With respect to each entity in a space, SCT partitions the space into a number of small unit cells and gives each cell a unique chromatic code. The spatial topological relationships among these cells are able to be represented and computed by their codes. Based on the different statistical rules on cellular codes, cells could be merged into larger clusters, such as a variety of Voronoi diagrams. Moreover, because cells are coded with the same data structure, it is easy to store and manage these codes in relational database, and then any spatial analytical operation could be realized simply by structured queries. SCT establishes a basic framework that not only provides a theoretical tool for spatial analysis and computations but also helps us to understand the space-entity relationship insightfully.
\end{abstract}

Keywords: spatial tessellation; spatial analysis; spatial topology; Voronoi diagrams

\section{Introduction}

To represent geographical entities and phenomena, there are two conceptually spatial data models, object- and fieldbased, serving in spatial analysis (Goodchild 1992, Lo and Yeung 2002). As one type of field-based model, spatial tessellation is an important approach to analyze geographical patterns and dynamics. In natural landscape, regions are divided into patches, corridors, and matrices (Dramstad et al. 1996). In human geography, countries and cities are divided into land lots, urban blocks, and social communities. Numerous data structures and models have been developed to represent and characterize spatial tessellations. The raster data model is actually the most well-known spatial tessellation that regularly partitions space into many minimum indivisible grids (Longley et al. 2005). The raster model is a nonentity model. It means that even without entities, the pure space could still be divided into grids. A low-resolution grid may represent a mixture of space and many entities, whereas many high-resolution grids may together represent the same space or one entity. The coordinates of a raster grid like $(x, y)$ or $(i, j)$ only indicate spatial locations. We are able to do spatial computations based on their coordinates but it is difficult to represent each subregion and entity uniquely and individually. Compared with the raster model, Voronoi tessellations are more associated with entities. They subdivide space into many irregular Voronoi regions with respect to the entities in the space (Okabe et al. 1992, Gold 1994). In Voronoi tessellations, the connections between spatial regions and entities are limited and too specific. For example, one Voronoi region is often assigned to one entity as its 'region of influence' or 'proximal region' (Aurenhammer 1991, Laurini and Thompson 1992), and therefore each Voronoi region could be marked by the entity's name such as 'Boston' or (a, b, c, d). However, we cannot do any mathematical operations on these name strings, for example, computing the distance between two regions. In short, the raster model is good at spatial computation and Voronoi diagrams focus much on entity representation. We may need a new model combining the two important aspects of geographical analysis.

Besides representing entities and space, spatial data models also serve to represent spatial relations between different real-world entities. Based on either object- or field-based data models, a variety of theories, models, and methods have been introduced from different perspectives. Region connection calculus (RCC) models are based on Clarke's calculus of individuals (Cohn 1992, Randell et al. 1992). The 4-I (intersection), 9-I, and Voronoi-based 9-I models consider the set operations between the interior, exterior, and boundaries of entities (Egenhofer and Franzosa 1991, Egenhofer and Herring 1991, Chen et al. 2001). Some scholars used spatial direction relations to reason topological relations (Frank 1996, Papadias and

*Corresponding author. Email: qyu@geo.umass.edu

ISSN 1947-5683 print/ISSN 1947-5691 online

(C) 2010 Taylor \& Francis

DOI: $10.1080 / 19475683.2010 .539983$

http://www.informaworld.com 
Theodorodis 1997), some employed regular grids to reason spatial relations (Kovalevsky 1989, Winter 1995), and some studied fuzzy spatial relation models such as the egg-yolk representation (Lehmann and Cohn 1994, Schneider 1999, Roy and Stell 2001). In addition, for spatial index and computation in geographic information system (GIS), many concrete data structures have been proposed, such as R-trees, pyramid-trees, and Hilbert curves (Guttman 1984, Faloutsos and Roseman 1989, Berchtold et al. 1998). However, no matter what models and structures are employed to represent spatial relations, in practice, most of the spatial reasoning, computation, and analysis are based on the spatial locations of geographical entities, which are featured as points, lines, and polygons, and are organized as coordinates, nodes, and arcs in spatial database. As a result, all of those traditional location-based spatial relation models often lead to some extent of complexity, because they involve complicated geometric computation using spatial coordinates.

In this study, we proposed a new spatial tessellation model - spatial chromatic tessellation (SCT) - and mainly focus on the so-called arranged chromatic diagram (ACD), a most common form of SCT. An ACD partitions space into a number of small irregular regions with respect to entities in the space. These irregular regions are called 'cells' in the terminology of computational geometry (O'Rourke 1998). Distinguished from the above-mentioned spatial data models and tessellations, ACDs assign each region a meaningful and distinct chromatic code as its inherent attribute. The chromatic codes, derived from an entity's attributes, could be thought of as the analogy of genetic codes that contain plentiful information and implication. One of the most significant points is that chromatic codes imply spatial topological relations and consequently are rather easy to reason, analyze, and compute without involving spatial coordinate calculation. In ACDs, cellular chromatic codes play a basic and critical role. Based on these codes, cells could be statistically merged into clusters oriented to different geographical applications.
The rest of this article is organized in the following manner. Section 2 describes how to partition space and assign chromatic codes and gives some basic concepts and properties of ACD. In Section 3, we discuss about the relationships between spatial topology and cellular or cluster chromatic codes. Section 4 demonstrates how to use statistical rules to merge cells into clusters and also shows the connections between ACDs and Voronoi diagrams. For better understanding of $\mathrm{ACD}$, the last section makes a conclusion and discusses related issues, further topics, as well as some implications.

\section{Spatial chromatic tessellation}

As a type of SCT, ACD's basic structure is quite straightforward. Here, we only introduce the simplest ACD. At first, a pair of entities induces one line and hence divides the space into two parts, which is the same way as in the abstract Voronoi diagrams to induce two half-planes (Klein 1989). Then an amount of entities will induce numerous lines and subdivisions. In computational geometry, such structures are equivalent to the arrangements of lines (O'Rourke 1998). Let $\boldsymbol{P}=\left\{p_{1}, p_{2}, \ldots, p_{n}\right\}$ be a set of $n$ entities in the plane, and each entity has a unique color as its index. Their colors comprise a color index set $\boldsymbol{C}=\left\{c_{1}, c_{2}, \ldots, c_{n}\right\}$. For every pair of entities, for instance, $p_{1}$ (blue) and $p_{2}$ (green) in Figure $1 \mathrm{a}$, there is a line $l\left(p_{1}, p_{2}\right)$ (or $l_{1,2}$ for short), called the dyed-boundary, partitioning the plane into two half-planes $\boldsymbol{H}\left(p_{1}, p_{2}\right)$ and $\boldsymbol{H}\left(p_{2}, p_{1}\right)$. The two half-planes are dyed the colors of corresponding entities respectively, that is, $\boldsymbol{H}\left(p_{1}, p_{2}\right)$ is dyed blue and $\boldsymbol{H}\left(p_{2}, p_{1}\right)$ green. All dyed-boundaries compose an arrangement of lines in which every face is dyed colors from the color index set. The face is also called a cell. Cells are bounded by edges (O'Rourke 1998) and are called closed if they are fully bounded or open if partly bounded. Figure $1 \mathrm{~b}$ shows the examples of open and closed cells induced by three entities. The diagram composed by all cells is called the ACD. Our subsequent study and analysis are restricted to the ordinary ACD (OACD), where entities

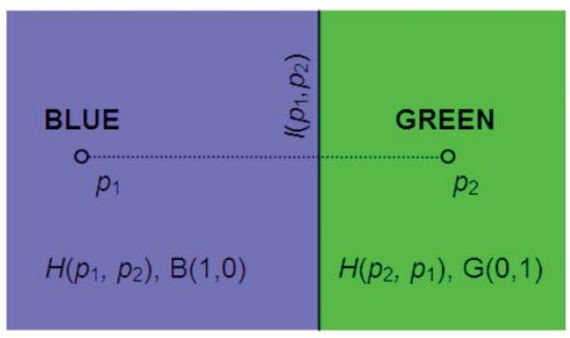

(a)

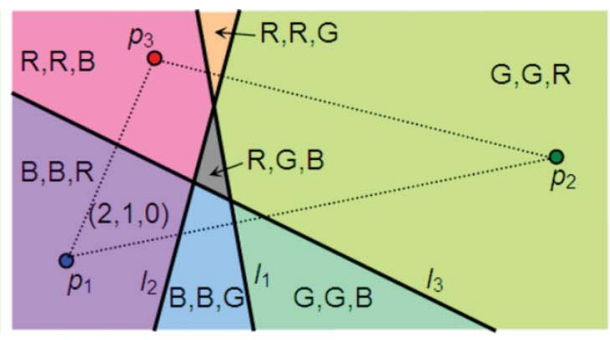

(b)

Figure 1. Partition and dye the space. (a) For two entities: Two half-planes are labeled (B) and $(\mathrm{G})$ and assigned codes $(1,0)$ and $(0,1)$, respectively. (b) For three entities: It consists of 7 cells -6 are open and 1 in the center is closed. The cell's label $(\mathrm{B}, \mathrm{B}, \mathrm{R})$ or code $(2,1,0)$ means with respect to three dyed-boundaries $l_{1}\left(p_{1}, p_{2}\right), l_{2}\left(p_{2}, p_{3}\right), l_{3}\left(p_{3}, p_{1}\right)$ that the cell is dyed blue twice (to $l_{1}$ and $l_{3}$ ), red once (to $\left.l_{2}\right)$, and no green. The special cell with color gray $(\mathrm{R}, \mathrm{G}, \mathrm{B})$ is called competition triangle. (Colour versions of all figures available online.) 
are point objects and dyed-boundaries are always perpendicular bisectors between two entities.

If $n$ entities in $\boldsymbol{P}$ induce a simple arrangement of dyedboundaries, which means every pair of dyed-boundaries meet exactly in one point and no three lines meet in a point, then the number of dyed-boundaries $L(n)$ and cells $D(n)$, respectively, are

$$
L(n)=\frac{1}{2} n^{2}-\frac{1}{2} n
$$

and

$$
D(n)=\frac{1}{8} n^{4}-\frac{1}{4} n^{3}+\frac{3}{8} n^{2}-\frac{1}{4} n+1
$$

If the dyed-boundaries are always the perpendicular bisectors, then

$$
D(n)=\frac{1}{8} n^{4}-\frac{5}{12} n^{3}+\frac{7}{8} n^{2}-\frac{7}{12} n+1
$$

With respect to each dyed-boundary $l_{i, j}$, a cell is always dyed a color either $c_{i}$ or $c_{j}$, therefore it contains $L(n)$ colors. A cell is denoted by $\left(c_{1}, c_{2}, \ldots, c_{L(n)}\right), c_{i} \in \boldsymbol{C}$, where $c_{i}$ is called the color component of the cell. For example, in Figure $2 \mathrm{a}, \zeta_{1}(1$, $1,1,2,4,4)$, with six color components, indicates that with respect to each dyed-boundary $l_{1,2}, l_{1,3}, l_{1,4}, l_{2,3}, l_{2,4}, l_{3,4}$, the cell is dyed six times with color $c_{1}, c_{1}, c_{1}, c_{2}, c_{4}, c_{4}$, respectively. Note that to compute the color components, dyed-boundaries need to predefine an order. In OACD, we use the order such as $l_{1,2}, l_{1,3}, l_{1,4}, l_{2,3}, l_{2,4}, l_{3,4}$, which can be defined as $l_{i, j}, i=1,2, \ldots, n-1$, and $j=i+1, i+2, \ldots, n$.

All cells derived from the entity set $\boldsymbol{P}$ form the ACD of $\boldsymbol{P}$, denoted by $\boldsymbol{A}(\boldsymbol{P})$,

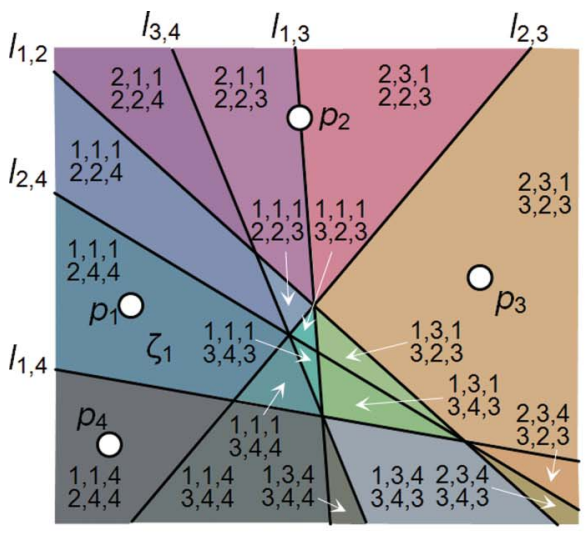

(a)

$$
\boldsymbol{A}(\boldsymbol{P})=\bigcup_{i=1}^{D(n)} \zeta_{i}
$$

A cell is dyed $L(n)$ times but only $n$ colors are available for dyeing, and when $n>3, L(n) \geq n$, so some colors must be dyed into a cell repeatedly. Therefore, a cell $\zeta$ could be expressed as $\zeta\left(\left[c_{1}, t_{1}\right],\left[c_{2}, t_{2}\right], \ldots,\left[c_{n}, t_{n}\right]\right), c_{i} \in \boldsymbol{C}$, where $\left[c_{i}, t_{i}\right]$ is called the color vector (denoted by $\lambda$ ) of the cell. $t_{i}$ indicates how many times the cell is dyed by $c_{i}$. We call $c_{i}$ the hue (denoted by $H\left(\lambda_{i}\right)$ ) and $t_{i}$ the saturation (denoted by $S$ $\left.\left(\lambda_{i}\right)\right)$ of a color vector $\lambda_{i}$. A cell $\zeta$ 's $i$ th color vector is denoted by $\zeta[i]=\lambda_{i}$ and then the cell is further denoted by $\zeta\left(\lambda_{1}, \lambda_{2}\right.$, $\left.\ldots, \lambda_{n}\right)$. For any given cells, their hues $c_{1}, c_{2}, \ldots, c_{n}$ are always the same but the saturations are variables, so we omit the hue and express a cell as $\zeta\left(t_{1}, t_{2}, \ldots, t_{n}\right)$, where the arrangement of $t_{1}, t_{2}, \ldots, t_{n}$ is called the chromatic code of the cell. For example, in Figure $2 b$, the cell $\zeta_{1}(1,1,1,2,4,4)$ is rewritten as $\zeta_{1}(3,1,0,2)$ with chromatic code $(3,1,0,2)$.

For any entity $p_{i}$, in total it induces $n-1$ dyedboundaries with the rest of $n-1$ entities in $\boldsymbol{P}$. If with respect to every induced dyed-boundary, a cell is always dyed $c_{i}$, then the maximum $t_{i}$ is $n-1$; on the contrary, if it is never dyed $c_{i}$, then the minimum $t_{i}$ is 0 , therefore we obtain the following constraint.

Given an ordinary $\boldsymbol{A}(\boldsymbol{P})$, for $\zeta \in \boldsymbol{A}(\boldsymbol{P})$ and $\lambda=\zeta[i]$,

$$
0 \leq S[\lambda] \leq n-1
$$

namely, $0 \leq \mathrm{t} \leq n-1$.

The purpose of partition and dyeing is to discretize space into many small fundamental elements, which hold the entity's attributes as their own attributes. All cellular chromatic codes of an entity set are arranged as a full-color table (see Table 1) for structural storage in a relational database.

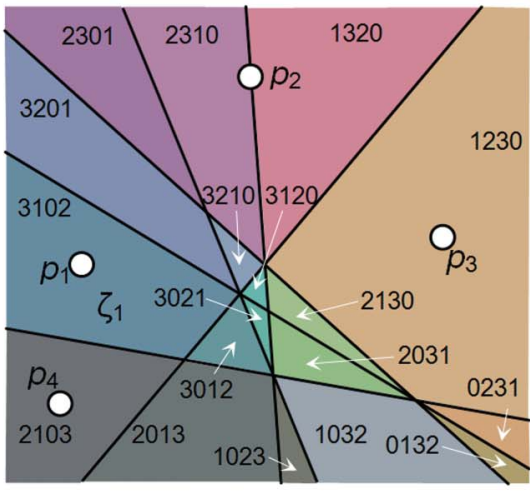

(b)

Figure 2. An ACD generated by four entities. (a) Cells are labeled by their color components. (b) Cells are labeled by their arranged chromatic codes. This ACD contains 18 cells, in which 6 cells are closed and 12 cells are open. 
Table 1. The full-color table of the ACD in Figure 2.

\begin{tabular}{|llllllllllllllllllll}
\hline & $\zeta_{1}$ & $\zeta_{2}$ & $\zeta_{3}$ & $\zeta_{4}$ & $\zeta_{5}$ & $\zeta_{6}$ & $\zeta_{7}$ & $\zeta_{8}$ & $\zeta_{9}$ & $\zeta_{10}$ & $\zeta_{11}$ & $\zeta_{12}$ & $\zeta_{13}$ & $\zeta_{14}$ & $\zeta_{15}$ & $\zeta_{16}$ & $\zeta_{17}$ & $\zeta_{18}$ \\
$\boldsymbol{\lambda}_{1}$ & 3 & 3 & 3 & 3 & 3 & 3 & 2 & 2 & 1 & 1 & 2 & 2 & 1 & 0 & 0 & 2 & 2 & 1 \\
$\boldsymbol{\lambda}_{2}$ & 1 & 2 & 2 & 1 & 0 & 0 & 3 & 3 & 3 & 2 & 1 & 0 & 0 & 2 & 1 & 1 & 0 & 0 \\
$\boldsymbol{\lambda}_{3}$ & 0 & 0 & 1 & 2 & 2 & 1 & 0 & 1 & 2 & 3 & 3 & 3 & 3 & 3 & 3 & 0 & 1 & 2 \\
$\lambda_{4}$ & 2 & 1 & 0 & 0 & 1 & 2 & 1 & 0 & 0 & 0 & 0 & 1 & 2 & 1 & 2 & 3 & 3 & 3 \\
\hline
\end{tabular}

Notes: One row represents an entity and one column represents a cell. The number inside a grid is $\lambda[s]$, indicating how many times an entity's color is dyed into the cell. This table could be alternatively transposed by interchanging its rows and columns.

\section{Spatial topology between cells and clusters}

The union of $n$ cells $\zeta_{1}, \zeta_{2}, \ldots, \zeta_{n}$ is called an $n$-cell cluster, denoted by $\xi\left\{\zeta_{1}, \zeta_{2}, \ldots, \zeta_{n}\right\}$, and in most cases, $n>1$ (one cell could be taken as 1 -cell cluster). These $n$ cells are called elementary cells of the cluster. It is an interesting property that spatial relations between cells and clusters can be derived by simple primary algebraic operations on chromatic codes. First, we define the operations of cellular chromatic union and intersection.

Given two cells $\zeta_{1}\left(t_{11}, t_{12}, \ldots, t_{1 n}\right)$ and $\zeta_{2}\left(t_{21}, t_{22}, \ldots\right.$, $\left.t_{2 n}\right)$, their chromatic union $(+) /$ intersection( $(-)$ are defined, respectively, by

$$
\zeta_{1} \pm \zeta_{2}=\left(t_{11} \pm t_{21}, t_{12} \pm t_{22}, \ldots, t_{1 n} \pm t_{2 n}\right)
$$

For example, $\zeta_{1}(3,2,1,0)+\zeta_{2}(3,1,2,0)=\xi\left\{\zeta_{1}\right.$, $\left.\zeta_{2}\right\}=\xi(6,3,3,0)$.

Because a cell always has $n$ color vectors, the above two operations thus could be further similarly extended to the union/intersection between a cell and a cluster, a cluster and a cluster, and among more than two cells and/or clusters. For the example of two clusters in Figure $4, \xi_{1}(6,3,3,0)+\xi_{2}(5$, $5,2,0)=\xi\left\{\xi_{1}, \xi_{2}\right\}=\xi(11,8,5,0)$.

Note that the chromatic union/intersection is only the operation of chromatic codes. The results of chromatic union/intersection may not be exactly equal to those of the corresponding spatial or set union/intersection. For example, if a cell unions with itself, then spatially it returns itself, but chromatically it returns a cell whose saturations are doubled.

Given two clusters $\xi_{1}\left(t_{11}, t_{12}, \ldots, t_{1 n}\right)$ and $\xi_{2}\left(t_{21}, t_{22}, \ldots\right.$, $\left.t_{2 n}\right)$, if $t_{11}=t_{21}, t_{12}=t_{22}, \ldots, t_{1 n}=t_{2 n}$, then $\xi_{1}$ and $\xi_{2}$ are called the equicolor and denoted by $\xi_{1}=\xi_{2}$.

The first property is that there are no equicolor cells in OACDs, that is, $\forall \zeta_{i}, \zeta_{j} \in \boldsymbol{A}(\boldsymbol{P})$ and $i \neq j$ :

$$
\zeta_{i} \neq \zeta_{j}
$$

The proof of this property is shown in the Appendix. Therefore, chromatic code could be taken as cellular unique identifier. The proof of Lemma 1 (see Appendix) also shows that all chromatic codes are permutations of a basic code set $\{0,1, \ldots, n\}$, which is called the primary chromatic code of cells or clusters.

The second interesting possible property is that the spatial topological relations between two cells are uniquely determined by a number, the transition number. Transition number between the two clusters $\xi_{1}\left(t_{11}, t_{12}, \ldots, t_{1 n}\right)$ and $\xi_{2}\left(t_{21}, t_{22}, \ldots, t_{2 n}\right)$ is denoted by $e\left(\xi_{1}, \xi_{2}\right)$, computed by

$$
e\left(\xi_{1}, \xi_{2}\right)=\sum_{i=1}^{n}\left|t_{1 i}-t_{2 i}\right|
$$

and shortly expressed as $e_{\xi 1}, \xi_{2}$ or $e_{1,2}$.

For an $n$-cell cluster $\xi\left\{\zeta_{1}, \zeta_{2}, \ldots, \zeta_{n}\right\}$, its interior transition number $E(\xi)$ is defined as the sum of transition numbers of all pairs of its elementary cells, that is,

$$
E(\xi)=\frac{1}{2} \sum_{i=1}^{n} \sum_{j=1}^{n} e\left(\zeta_{i}, \zeta_{j}\right), \zeta_{i}, \zeta_{j} \in \xi
$$

A cluster with interior transition number $k$ is called a $k$-transferred cluster.

In Figure 3a1, we take $\zeta_{4}$, the pink cell, as an example and observe the following spatial topology (the cellular indices, chromatic codes, and transition numbers are referred to in Figure 3b-d, respectively: $\zeta_{3}, \zeta_{5}, \zeta_{11}$ (the orange cells) share $\zeta_{4}$ with a common edge, so that they could be taken as its nearest neighbors, and in these cases, $e=2 ; \zeta_{1}, \zeta_{2}, \zeta_{6}, \zeta_{8-10}, \zeta_{12}$ (the green cells) are the second nearest neighbors, connecting $\zeta_{4}$ with only a common vertex, and in these cases, $e=4$; the spatial topological relations between $\zeta_{4}$ and $\zeta_{7}, \zeta_{13-18}$ (the blue cells) are disconnected, and in these cases, $e=6$. Actually, it is easy to find out a possible property that 'for two cells, the larger $e$ they have, the further they are.' Note here the 'further' means topological relations instead of spatial distance. In this sense, we call transition number the chromatic distance between two clusters.

We checked all six closed cells in Figure $3 \mathrm{a} 1$ and they keep the same property. Open cells, however, do not entirely follow the property. We think it is due to the boundary effect 


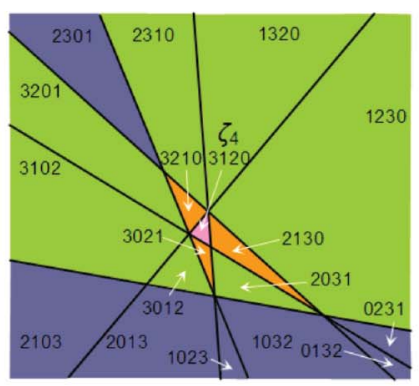

(a1)

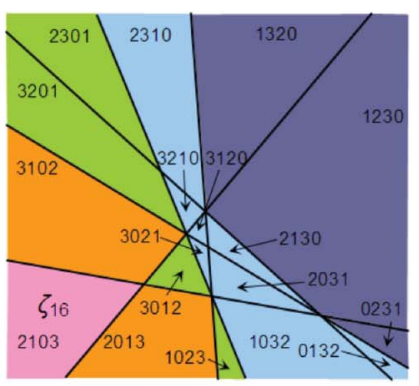

(a2)

\begin{tabular}{|c|c|c|c|c|c|c|}
\hline$\zeta_{4}$ & $\zeta_{3}$ & $\zeta_{5}$ & $\zeta_{11}$ & $e_{4,3}$ & $e_{4,5}$ & $e_{4,1}$ \\
\hline 3 & 3 & 3 & 2 & 0 & 0 & +1 \\
\hline 1 & 2 & 0 & 1 & -1 & +1 & 0 \\
\hline 2 & 1 & 2 & 3 & +1 & 0 & -1 \\
\hline \multirow[t]{2}{*}{0} & 0 & 1 & 0 & 0 & -1 & 0 \\
\hline & Tran & & oer: & 2 & 2 & 2 \\
\hline
\end{tabular}

(b) The 1st nearest neighbors, connected by a common edge

\begin{tabular}{ccccccccccccccc}
\hline$\zeta_{4}$ & $\zeta_{1}$ & $\zeta_{2}$ & $\zeta_{6}$ & $\zeta_{8}$ & $\zeta_{9}$ & $\zeta_{10}$ & $\zeta_{12}$ & $e_{4,1}$ & $e_{4,2}$ & $e_{4,6}$ & $e_{4,8}$ & $e_{4,9}$ & $e_{4,10}$ & $e_{4,12}$ \\
\hline 3 & 3 & 3 & 3 & 2 & 1 & 1 & 2 & 0 & 0 & 0 & +1 & +2 & +2 & +1 \\
1 & 1 & 2 & 0 & 3 & 3 & 2 & 0 & 0 & -1 & +1 & -2 & -2 & -1 & +1 \\
2 & 0 & 0 & 1 & 1 & 2 & 3 & 3 & +2 & +2 & +1 & +1 & 0 & -1 & -1 \\
0 & 2 & 1 & 2 & 0 & 0 & 0 & 1 & -2 & -1 & -2 & 0 & 0 & 0 & -1 \\
\hline
\end{tabular}

(c) The 2nd nearest neighbors, connected by a common vertex

\begin{tabular}{ccccccccccccccc}
\hline$\zeta_{4}$ & $\zeta_{7}$ & $\zeta_{13}$ & $\zeta_{14}$ & $\zeta_{15}$ & $\zeta_{16}$ & $\zeta_{17}$ & $\zeta_{18}$ & $e_{4,7}$ & $e_{4,13}$ & $e_{4,14}$ & $e_{4,15}$ & $e_{4,16}$ & $e_{4,17}$ & $e_{4,18}$ \\
\hline 3 & 2 & 1 & 0 & 0 & 2 & 2 & 1 & +1 & +2 & +3 & +3 & +1 & +1 & +2 \\
1 & 3 & 0 & 2 & 1 & 1 & 0 & 0 & -2 & +1 & -1 & 0 & 0 & +1 & +1 \\
2 & 0 & 3 & 3 & 3 & 0 & 1 & 2 & +2 & -1 & -1 & -1 & +2 & -1 & 0 \\
0 & 1 & 2 & 1 & 2 & 3 & 3 & 3 & -1 & -2 & -1 & -2 & -3 & -3 & -3 \\
\hline
\end{tabular}

(d) The 3rd nearest neighbors, disconneted

Figure 3. Topological relations between cells and transition numbers. In (a1) and (a2), cells with same colors have same transition numbers to the pink cell $\zeta_{4}(3,1,2,0)$ or $\zeta_{16}(2,1,0,3)$. (a1) shows the spatial relations between $\zeta_{4}$ and other cells. (a2) shows an open cell $\zeta_{16}$ and other cells with transition numbers $2,4,6,8$ (dyed with the same color, respectively). (b)-(d) show the transition numbers corresponding to their spatial relations.

because there are always some points lying in the convex hull of a given point set in space. For example, in Figure 3a2, with respect to $\zeta_{16}(2,1,3,0)$, yellow, green, light blue, and dark blue cells have transition numbers $2,4,6$, and 8 , respectively. Although $e=2$ still corresponds to the nearest neighbors, the correspondences between spatial relations and transition numbers when $e=4,6,8$ are not as explicit and distinct as those in the cases of closed cells. For instance, cells $\zeta(2,3,0,1)$ and $\zeta_{16}$ are disconnected but they have a small transition number 4. An approach to handle this problem is extending $\mathrm{ACD}$ to three-dimensional space (imaging all points located on a sphere, a dyed-boundary turns to a great circle of the sphere, and a half-plane turns to a hemispheric surface), then all open cells are closed (e.g., $\zeta(2,3,0,1)$ and $\zeta_{16}$ are actually connected by a common vertex on the other hemisphere). An alternative approach to solve this problem is to calculate the shortest path (see the Appendix). The above two approaches are quite complicated and we do not intend to give full details here. Nevertheless, in a general geographical space $\Re^{2}$ with a great amount of entities, most of the cells are closed and only a few marginal cells are open but not our focus in this study. 
The above properties between cells still work for clusters. First, we consider 2-cell clusters are totally $D(n)$ combinations of unions of two cells. For these 2-cell clusters, they may have interior transition number $2,4,6,8$, and so on. We call the cluster with the minimum interior transition number the minimum transferred or simple cluster. For two cells connected with a common edge, they unite into a simple 2-cell cluster, that is, a 2-transferred cluster.

In OACDs, the definition of simple clusters is the same as the simple polygons (Okabe et al. 1992). Table 2 shows possible chromatic codes of all simple 2-cell clusters generated from an entity set $\boldsymbol{P}$ with four entities. The correspondences between spatial relation and transition number of simple 2-cell clusters are similar to those in the 1-cell clusters. Figure 4 demonstrates an example of 2-cell's spatial relations and their associated transition numbers. We can still observe that for two 2-cell clusters, the larger transition number they have, the topologically further they are.

Table 2 shows that arranged chromatic codes of all simple 2-cell clusters are still always distinct but come from one of the cluster primary chromatic codes $\{0,2,5,5\},\{0,3,3,6\}$, $\{1,1,4,6\}$. In this OACD, the cellular primary code is $\{0,1$, $2,3\}$, and when a cell transfers one number, there are only three alternatives to keep primary chromatic code unchanged: from 0 to 1,1 to 2 , or 2 to 3 . Table 3 shows why such three primary codes arise. In the same way, we computed that for 4-transferred 2-cell clusters, their primary codes are always $\{0,3,4,5\},\{0,4,4,4\},\{1,1,5,5\},\{1,2$, $3,6\},\{2,2,2,6\}$, distinct from those of the simple clusters. For the 6-transferred, their primary codes are $\{1,2,4,5\}$, $\{1,3,3,5\},\{1,3,4,4\},\{2,2,3,5\},\{2,3,3,4\}$, and the 8 transferred are $\{2,2,4,4\},\{2,3,3,4\},\{3,3,3,3\}$. All of these primary chromatic codes are distinct from each other, except the 6- and 8-transferred sharing the same code $\{2,3,3,4\}$.

For simple 3-cell clusters, they have the same property. Table 4 lists all the chromatic codes of 3-cell simple clusters, which should bear interior transition number 8 . Their codes are still distinct with primary chromatic codes $\{1,3,5,9\},\{1,2,7,8\}$, or $\{0,4$, $6,8\}$. We hope to find a general expression to represent the spatial relations between all simple clusters by their transition numbers. Here, we just report the above observations found in OACDs and would like it to remain as an open problem and hope it can be rigorously solved in mathematics.

Table 2. 2-Cell simple clusters with primary chromatic codes $\{0$, $2,5,5\},\{0,3,3,6\}$, and $\{1,1,4,6\}$.

\begin{tabular}{lccc}
\hline$\zeta_{1}$ & $\zeta_{2}$ & $e_{1,2}$ & $\zeta_{1}+\zeta_{2}$ \\
\hline 3210 & 3201 & 2 & 6411 \\
3120 & 3210 & 2 & 6330 \\
3102 & 3201 & 2 & 6303 \\
3120 & 3021 & 2 & 6141 \\
3102 & 3012 & 2 & 6114 \\
3021 & 3012 & 2 & 6033 \\
3210 & 2310 & 2 & 5520 \\
3201 & 2301 & 2 & 5502 \\
3120 & 2130 & 2 & 5250 \\
3102 & 2103 & 2 & 5205 \\
3021 & 2031 & 2 & 5052 \\
3012 & 2013 & 2 & 5025 \\
2310 & 2301 & 2 & 4611 \\
2130 & 2031 & 2 & 4161 \\
2103 & 2013 & 2 & 4116 \\
2310 & 1320 & 2 & 3630 \\
2130 & 1230 & 2 & 3360 \\
2031 & 1032 & 2 & 3063 \\
2013 & 1023 & 2 & 3036 \\
1320 & 1230 & 2 & 2550 \\
1032 & 1023 & 2 & 2055 \\
1230 & 0231 & 2 & 1461 \\
1032 & 0132 & 2 & 1164 \\
0231 & 0132 & 2 & 0363 \\
\hline & & &
\end{tabular}

Note: Each cluster's chromatic code is unique. (a)

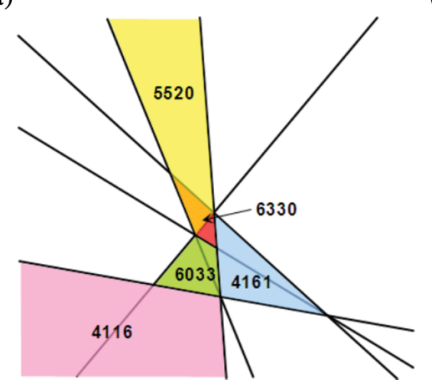

(b)

\begin{tabular}{|c|c|c|c|}
\hline$\xi_{1}$ & $\xi_{2}$ & $e_{1,2}$ & Spatial topological relation \\
\hline 6330 & 5520 & 4 & Connected by 1 common cell \\
\hline 6330 & 6033 & 6 & Connected by 1 common edge and 1 vertex ${ }^{a}$ \\
\hline 6330 & 4161 & 8 & Connected by 1 common edge $b$ \\
\hline 6033 & 4161 & 8 & Connected by 1 common edge \\
\hline 6033 & 4116 & 8 & Connected by 1 common edge \\
\hline 4116 & 4161 & 10 & Connected by 1 common vertex \\
\hline 6330 & 4116 & 12 & Disconnected \\
\hline 4116 & 5520 & 12 & Disconnected \\
\hline
\end{tabular}

Figure 4. Examples for transition numbers and spatial relations between 2-cell clusters. a: The relation between the red cluster $\xi(6330)$ and the green $\xi(6033)$. Their common vertex comes from their elementary cells 3210 and 3012 and the common edge comes from cells 3120 and 3021 (see Figure $2 \mathrm{~b}$ ). b: The relation between $\xi(6330)$ and the blue cluster $\xi(4161)$. They have no common vertex such as in a, because their elementary cells 3210 and 2031 are disconnected. In b, clusters' spatial relation is equivalent to be connected by a partial common cluster edge. 
Table 3. Three scenarios with transition number 2.

\begin{tabular}{lc}
\hline Transitions with $e=2$ & Primary codes of 2-cell clusters \\
\hline $0123(0 \leftrightarrow 1) \rightarrow 1023$ & $0123+1023=1146$ \\
$0123(1 \leftrightarrow 2) \rightarrow 0213$ & $0123+0213=0336$ \\
$0123(2 \leftrightarrow 3) \rightarrow 0132$ & $0123+0132=0255$ \\
\hline
\end{tabular}

Note: The formula in the first grid denotes when 0 and 1 exchange their places, 0123 transfers to 1023 .

\section{Interpretation of arranged chromatic codes}

An important function of ACD is that cells are able to be merged into a large cluster. This section introduces how to statistically interpret and integrate cellular chromatic codes.
In ACDs, cellular aggregation is based on their full-color tables. In relational database, a full-color table consists of columns and rows, representing cells and entities, respectively. Or inversely, we can use columns to represent entities and rows to represent cells. In terms of different statistics in full-color table, that is, the chromatic merging rules, cells could be merged into clusters hence forming new spatial tessellations or patterns.

\subsection{Entity-based merging rules}

The saturation of a color vector means how much influence an entity imposes on the cell. Then from an entity's

Table 4. 3-Cell simple clusters with interior transition number 8 and primary chromatic codes $\{0,4,6,8\},\{1,2,7,8\}$ and $\{1,3,5,9\}$.

\begin{tabular}{|c|c|c|c|c|c|c|c|}
\hline$\zeta_{1}$ & $\zeta_{2}$ & $\zeta_{3}$ & $e_{1,2}$ & $e_{1,3}$ & $e_{2,3}$ & $E(\xi)$ & $\zeta_{1}+\zeta_{2}+\zeta_{3}$ \\
\hline 1230 & 0231 & 0132 & 2 & 2 & 4 & 8 & 1593 \\
\hline 2031 & 1032 & 0132 & 2 & 2 & 4 & 8 & 3195 \\
\hline 1320 & 1230 & 0231 & 2 & 2 & 4 & 8 & 2781 \\
\hline 2130 & 1230 & 0231 & 2 & 2 & 4 & 8 & 3591 \\
\hline 2031 & 1032 & 1023 & 2 & 2 & 4 & 8 & 4086 \\
\hline 2103 & 2013 & 1023 & 2 & 2 & 4 & 8 & 5139 \\
\hline 3012 & 2013 & 1023 & 2 & 2 & 4 & 8 & 6048 \\
\hline 2130 & 2031 & 1032 & 2 & 2 & 4 & 8 & 5193 \\
\hline 3021 & 2031 & 1032 & 2 & 2 & 4 & 8 & 6084 \\
\hline 2310 & 1320 & 1230 & 2 & 2 & 4 & 8 & 4860 \\
\hline 3120 & 2130 & 1230 & 2 & 2 & 4 & 8 & 6480 \\
\hline 3210 & 2310 & 1320 & 2 & 2 & 4 & 8 & 6840 \\
\hline 3102 & 2103 & 2013 & 2 & 2 & 4 & 8 & 7218 \\
\hline 3021 & 3012 & 2013 & 2 & 2 & 4 & 8 & 8046 \\
\hline 3102 & 3012 & 2013 & 2 & 2 & 4 & 8 & 8127 \\
\hline 3120 & 2130 & 2031 & 2 & 2 & 4 & 8 & 7281 \\
\hline 3120 & 3021 & 2031 & 2 & 2 & 4 & 8 & 8172 \\
\hline 3210 & 2310 & 2301 & 2 & 2 & 4 & 8 & 7821 \\
\hline 3102 & 3201 & 2301 & 2 & 2 & 4 & 8 & 8604 \\
\hline 3210 & 3201 & 2301 & 2 & 2 & 4 & 8 & 8712 \\
\hline 3120 & 3210 & 2310 & 2 & 2 & 4 & 8 & 8640 \\
\hline 3120 & 3021 & 3012 & 2 & 2 & 4 & 8 & 9153 \\
\hline 3120 & 3210 & 3201 & 2 & 2 & 4 & 8 & 9531 \\
\hline 1032 & 0132 & 1023 & 2 & 4 & 2 & 8 & 2187 \\
\hline 2130 & 1230 & 2031 & 2 & 4 & 2 & 8 & 5391 \\
\hline 3021 & 3012 & 2031 & 2 & 4 & 2 & 8 & 8064 \\
\hline 3102 & 3012 & 2103 & 2 & 4 & 2 & 8 & 8217 \\
\hline 3102 & 3201 & 2103 & 2 & 4 & 2 & 8 & 8406 \\
\hline 3120 & 3021 & 2130 & 2 & 4 & 2 & 8 & 8271 \\
\hline 3120 & 3210 & 2130 & 2 & 4 & 2 & 8 & 8460 \\
\hline 2310 & 1320 & 2301 & 2 & 4 & 2 & 8 & 5931 \\
\hline 3210 & 3201 & 2310 & 2 & 4 & 2 & 8 & 8721 \\
\hline 3102 & 3201 & 3012 & 2 & 4 & 2 & 8 & 9315 \\
\hline 3120 & 3210 & 3021 & 2 & 4 & 2 & 8 & 9351 \\
\hline 1032 & 0231 & 0132 & 4 & 2 & 2 & 8 & 1395 \\
\hline 1032 & 2013 & 1023 & 4 & 2 & 2 & 8 & 4068 \\
\hline 2130 & 1320 & 1230 & 4 & 2 & 2 & 8 & 4680 \\
\hline 3012 & 2103 & 2013 & 4 & 2 & 2 & 8 & 7128 \\
\hline 3021 & 2130 & 2031 & 4 & 2 & 2 & 8 & 7182 \\
\hline 3201 & 2310 & 2301 & 4 & 2 & 2 & 8 & 7812 \\
\hline 3021 & 3102 & 3012 & 4 & 2 & 2 & 8 & 9135 \\
\hline 3210 & 3102 & 3201 & 4 & 2 & 2 & 8 & 9513 \\
\hline
\end{tabular}

Note: Each cluster's chromatic code is unique. 
perspective, the first rule is finding out the cells where one entity has the most dominance than the others.

(NEIGHBORHOOD, $\mathrm{R}_{1}$ ) For an entity $p_{i}$, merge all cells where its saturation $t_{i}$ is the maximum, that is,

$$
\xi\left(p_{i}, \mathrm{R}_{1}\right)=\left\{\zeta: t_{i}=n-1\right\}
$$

where $\xi\left(p_{i}, \mathbf{R}_{1}\right)$ is the cluster of $p_{i}$ with respect to Rule 1 (called the neighborhood rule and denoted by $\mathrm{R}_{1}$ ), and $\{\zeta$ : $\left.t_{i}=n-1\right\}$ is a set that contains all cells with property $t_{i}=n-1$. In this rule, $p_{i}$ is called the generator of the cluster. The maximum $t_{i}=n-1$ is obtained from Equation (5). Equation (10) is abbreviated and denoted by

$$
\xi\left(p_{i}, \mathrm{R}_{1}\right)=<n-1>
$$

Because every entity generates a cluster in this rule, the clusters generated from all entities compose a new diagram named neat ACD (NACD). For $\mathrm{R}_{1}$, its NACD is denoted by

$$
\boldsymbol{A}_{\mathrm{N}}\left(\boldsymbol{P}, \mathrm{R}_{1}\right)=\bigcup_{i=1}^{n} \xi\left(p_{i}, \mathrm{R}_{1}\right)
$$

In Table $5 \mathrm{a}, \mathrm{R}_{1}$ is illustrated by a rule-color table extracted from a full-color table. In this rule-color table, for every row, the cells with the same maximum saturation, that is, the grids with the same color, are merged into clusters, as in Figure 5a.

For an entity $p_{i}$, its cluster merged from $\mathrm{R}_{1}$ is equivalent to its ordinary Voronoi region, that is,

$$
\xi\left(p_{i}, \mathbf{R}_{1}\right)=V\left(p_{i}\right), p_{i} \in \boldsymbol{P}
$$

Because in OACD the dyed-boundary is a perpendicular bisector, the points in $\boldsymbol{H}\left(p_{i}, p_{j}\right)$ being dyed color $c_{i}$ indicate that $p_{i}$ is its nearer entity than $p_{j}$. For any cell in $\boldsymbol{A}(\boldsymbol{P})$, the saturation of $c_{i}$ being $n-1$ indicates that for all dyedboundaries induced by $p_{i}$, the cell are always dyed color $c_{i}$. Hence for any point in the cell, the $p_{i}$ is the nearest

Table 5. Rule-color tables of Rules 1-4 ((a)-(d)) and high-order Voronoi diagrams ((e) and (f)).

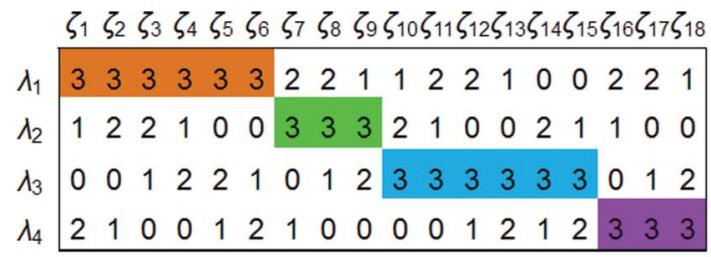

(a) Rule 1, NEIGHBORHOOD, $s=n-1$

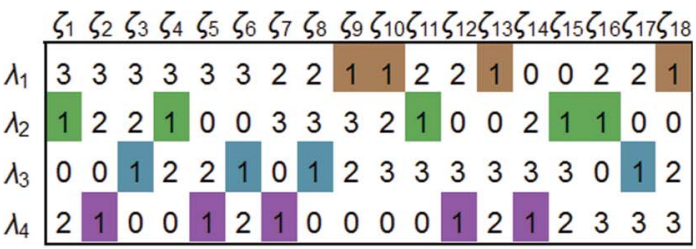

(c) Rule 3, $s=n-3$

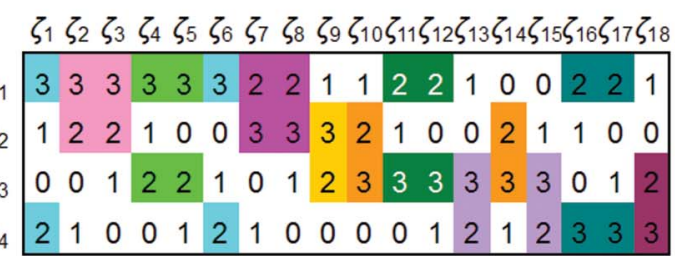

(e) Ordered order-2 Voronoi diagrams

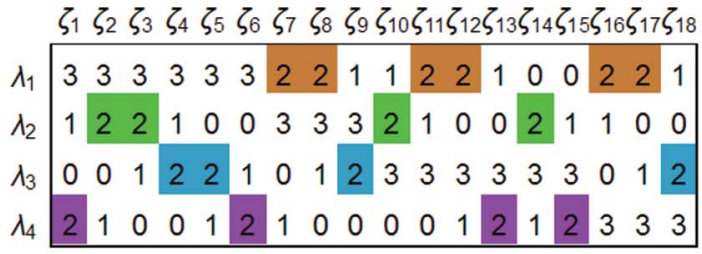

(b) Rule 2, $s=n-2$

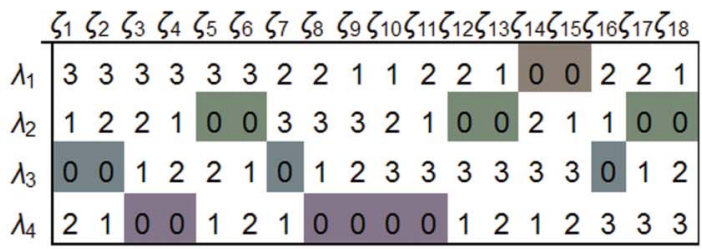

(d) Rule 4, $s=0$

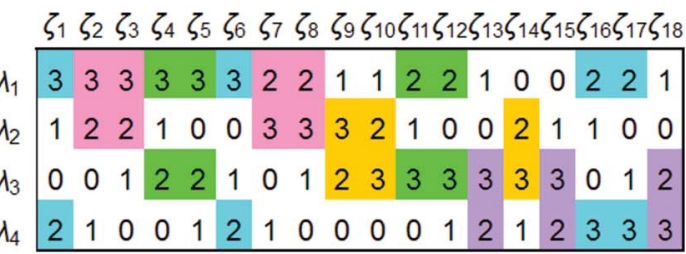

(f) Order-2 Voronoi diagrams

Note: In each table, cells marked with the same color are merged into one cluster. 


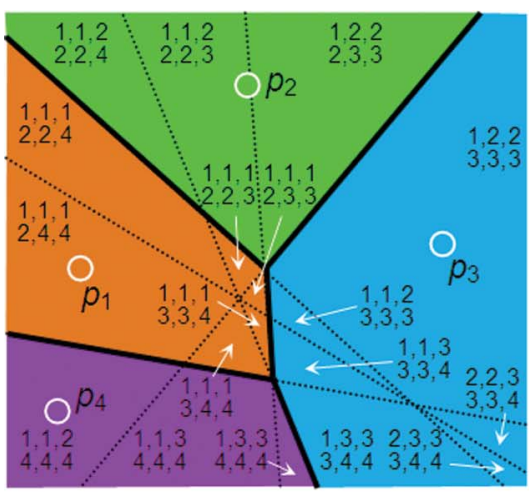

(a) Rule $1=\mathcal{V}(\boldsymbol{P})$

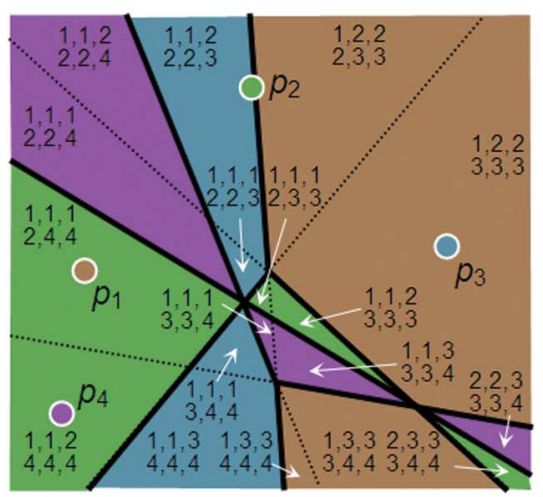

(c) Rule $3=\mathcal{V}^{[3]}(\boldsymbol{P})$

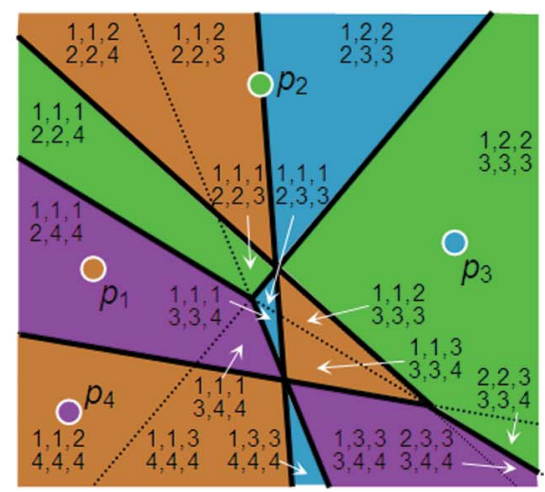

(b) Rule $2=\mathcal{V}^{[2]}(\boldsymbol{P})$

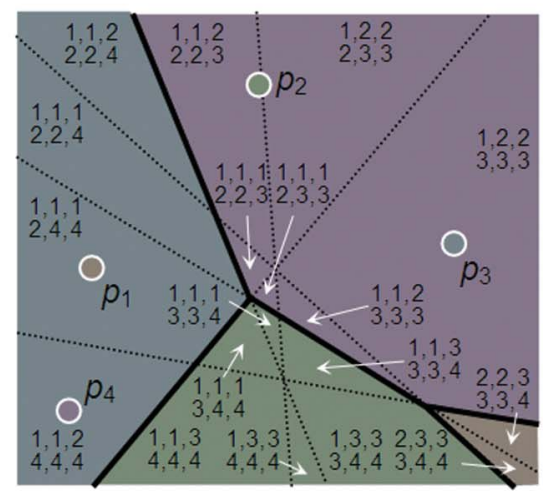

(d) Rule $4=\mathcal{V}_{\text {fp }}(\boldsymbol{P})$

Figure 5. NACDs generated from four entities in Rules 1-4.

entity among the $\boldsymbol{P}$. Also in $\mathrm{R}_{1}$, all the cells that satisfy the above property are merged into a cluster and such a clustered region is the same as the definition of Voronoi region, which consists of all the points at least as close to $p_{i}$ as to any other entities $p_{j}$.

Based on the above proof, we conclude that NACD in Rule 1 is equivalent to the ordinary Voronoi diagram, that is,

$$
\boldsymbol{A}_{\mathrm{N}}\left(\boldsymbol{P}, \mathrm{R}_{1}\right)=V(\boldsymbol{P})
$$

There are several other similar rules as follows:

$\left(\mathrm{R}_{2}\right)$ For an entity $p_{i}$, merge all cells where its saturation $t_{i}$ is the second largest, that is,

$$
\xi\left(p_{i}, \mathrm{R}_{2}\right)=<n-2>
$$

$\left(\mathrm{R}_{3}\right)$ For an entity $p_{i}$, merge all cells where its saturation $t_{i}$ is the third largest, that is,

$$
\xi\left(p_{i}, \mathrm{R}_{3}\right)=<n-3>
$$

$\left(\mathrm{R}_{4}\right)$ For an entity $p_{i}$, merge all cells where its saturation $t_{i}$ is 0 , that is,

$$
\xi\left(p_{i}, \mathrm{R}_{4}\right)=<0>
$$

$\mathrm{R}_{2}-\mathrm{R}_{4}$ are equivalent to the second-, third-nearest point, and furthest point Voronoi diagrams, respectively, that is, $\boldsymbol{A}_{\mathrm{N}}(\boldsymbol{P}$, $\left.\mathrm{R}_{2}\right)=\boldsymbol{V}^{[2]}(\boldsymbol{P}), \boldsymbol{A}_{\mathrm{N}}\left(\boldsymbol{P}, \mathrm{R}_{3}\right)=\boldsymbol{V}^{[3]}(\boldsymbol{P})$, and $\boldsymbol{A}_{\mathrm{N}}\left(\boldsymbol{P}, \mathrm{R}_{4}\right)=\boldsymbol{V}_{\mathrm{fp}}(\boldsymbol{P})$, respectively. Their rule-color tables and NACDs are shown in Table $5 \mathrm{~b}-\mathrm{d}$ and Figure $5 \mathrm{~b}-\mathrm{d}$.

From $R_{1}$ to $R_{4}$, the saturation decreases from the maximum to 0 . We organize rules like $R_{1}-R_{4}$ to a rule group that corresponds to the $k$ th-nearest point Voronoi diagrams:

$$
\mathrm{R}<0,1,2, \ldots, n>
$$


$\mathrm{ACD}$ is also easy to generate high-order $k$, for example, the ordered order-2 and order-2 Voronoi diagrams. To do so, their rules should be, respectively, as follows:

$$
\xi\left(\left(p_{i}, p_{j}\right), \mathrm{R}_{\text {ordered }-2}\right)=\left\{\zeta: t_{\mathrm{i}}=n-1 \cap t_{j}=n-2\right\}
$$

and

$$
\xi\left(\left(p_{i}, p_{j}\right), \mathrm{R}_{\text {order }-2}\right)=\left\{\zeta: t_{i}+t_{j}=2 n-3\right\}
$$

Their rule-color tables are given in Table 5e and f. In fact, from the perspective of ordering, for $n$ entities, when the order $k$ reaches its maximum $n$, OACDs could be hence regarded as the ordered highest-order (i.e., order- $n$ ) Voronoi diagrams, which are too trivial to be studied by most of the spatial analysis communities. In ordered highest-order Voronoi diagrams, the ordered $n$-orders may be the same as chromatic codes, because in ACDs their corresponding rule is

$$
\left.<t_{i}=0 \cap t_{j}=1 \cap \ldots \cap t_{n}=n-1\right\}
$$

Equation (21) is similar to the permutation of cellular primary chromatic codes. In this case, each cell is merged into itself due to the nonexistence of equicolor cells. Although OACDs and the ordered highest-order Voronoi diagrams seem identical in their appearance, they are actually different in essence. In OACDs, chromatic codes are quantitative characteristics rather than qualitative labels in ordered highorder Voronoi diagrams. For example, given 4 entities (labeled by A, B, C, D or $0,1,2,3$ ), in their ordered order-4 Voronoi diagrams, ' 3 ' ' in the order $(3,0,1,2)$ or ' $\mathrm{B}$ ' in the order $(\mathrm{B}, \mathrm{C}, \mathrm{A}, \mathrm{D})$ is just a symbol indicating that the nearest entity is labeled by ' 3 ' or ' $\mathrm{B}$,' but in OACDs, 3 in code $(3,0,1,2)$ is a number indicating the magnitude of influence imposed by the first entity.

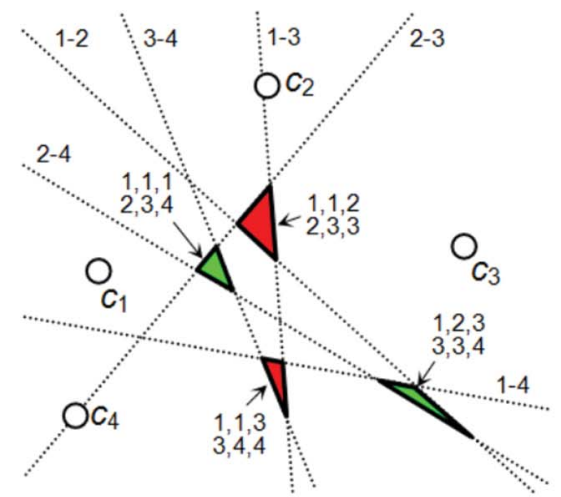

(a)
Chromatic codes provide not only a new perspective to generate and interpret Voronoi diagrams but also abundant information for more statistical merging. How to set merging rules depends on the addressed problem or a specific application. The statistics of chromatic codes are usually along two directions: entities and cells, namely, the rows and columns of a full-color table. Ordinary Voronoi diagrams are entity-based interpretations and they only involve with the maximum saturation. Other statistics, such as minimum, sum, average, and even complicated statistical distributions, are all possible variables to merge cells. In entitybased merging, each cluster is relevant to one or more generators, whereas in Section 4.2, we will introduce cellbased merging, which focuses on permutation characteristics of a chromatic code, regardless of who the generators are.

\subsection{Singular cells and their geographical significance}

In the OACD induced by 4 entities, the cellular chromatic codes are always the permutations of the primary chromatic code $\{0,1,2,3\}$. However, other primary chromatic codes, such as $\{0,2,2,2\},\{1,1,1,3\}$, and $\{1,1,2,2\}$, should be allowed at least theoretically. For $n$ entities, we call their primary chromatic code $\{0,1,2, \ldots, n\}$ the normal primary chromatic code and otherwise the singular primary chromatic codes. This subsection focuses on these singular cells and explains their geographical significance. In fact, the singular cells are degenerated into vertices in OACDs (see an example in Figure 6b). If dyed-boundaries are not bisectors, the singular codes will occur in ACDs (see Figure 6).

Chromatic codes indicate how much influence every entity could impact on a subregion. For instance, $p_{1}-p_{4}$ are the quarters of four gangs and they set their territories by roads. In this instance, gangs are entities located in $p_{1}-p_{4}$, roads are dyed-boundaries, and urban blocks bounded by roads are cells. From the geographical perspective, normal

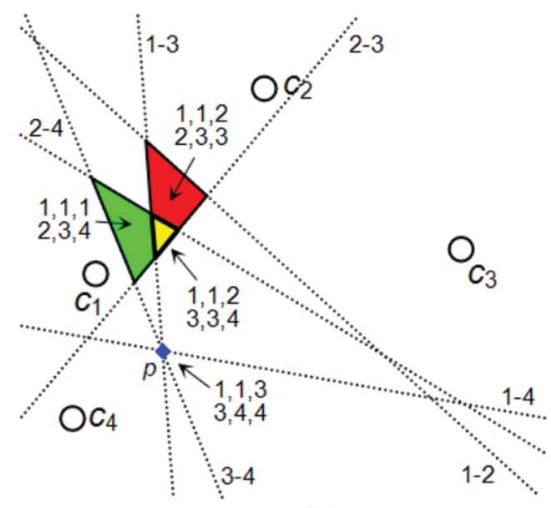

(b)

Figure 6. Singular cells in ACD. (a) Cases for $\{3,1,1,1\}$ (green cells) and $\{2,2,2,0\}$ (red cells). (b) Cases for $\{2,2,1,1\}$ (yellow cells) and a degenerated cell (blue vertex). 
primary chromatic code $\{0,1,2,3\}$ is an ordered distribution where the gangs' dominance is graded distinctively. $\{0$, $2,2,2\}$ is a uniform distribution where three gangs' dominance is balanced in a high level, so that the three gangs may compete for their territories. In $\{1,1,1,3\}$, three gangs are balanced in a low level although one gang has an outstanding dominance, so that the three gangs may either compete with each other in a low level or they are more likely inclined to cooperate to contend with the dominant gang. $\{1,1,2,2\}$ means two gangs are balanced in low level whereas the other two gangs are in the high. Because of the administration of public security, a police station may concern those singular cells where an open fight may happen, because they could be regarded as competitive or isolated regions never being exclusively dominated by any entities.

Primary chromatic codes could be rearranged to all possible chromatic codes. It is easy to know that the normal code $\{0,1,2,3\}$ generates 24 permutations, whereas $\{1,1$, $2,2\},\{0,2,2,2\}$, and $\{1,1,1,3\}$ generate 6,4 , and 4 permutations, respectively. This property indicates that in ACD most cells are with normal primary chromatic code $\{0$, $1,2,3\}$, occupying the most of the space. In these cells, each entity is orderly settled in an appropriate position and there are no entities in extreme dominance as in $\{1,1,1,3\}$ or competitive balance as in $\{0,2,2,2\}$.

Besides entity-based rules, cells could also be merged in cell-based rules. The cell-based rules only consider the statistics of cellular primary chromatic code without considering who the generator of a cluster is. Rule 5 is an example.

$\left(\mathrm{R}_{5}\right)$ Given an ACD generated by $n$ entities, merge all cells where the maximum saturation is $n-1$, that is,

$$
\xi\left(\mathrm{R}_{5}\right)=\{\zeta: \max (t)=n-1\}
$$

In this rule, all cells with primary chromatic codes $\{0,1,2$, $3\}$ or $\{1,1,1,3\}$ are merged into a cluster. If the ACD is an
OACD, because all cells have the same primary chromatic code $\{0,1,2,3\}$, we then obtain the whole space.

Furthermore, $\{0,0,3,3\}$ is also a possible singular code, but where is it? Actually, such cells do not exist in OACDs, that is,

$$
t_{i}=t_{j}=n-1 \text { or } t_{i}=t_{j}=0, ! \exists t_{i}, t_{j} \in \zeta, i \neq j
$$

Because $t_{i}=n-1$ indicates that with respect to the dyedboundary $l\left(p_{i}, p_{j}\right)$, the cell is always dyed $c_{i}$, whereas $t_{i}=n-1$ indicates that it is always dyed $c_{j}$ in the same way, but with respect to $l\left(p_{i}, p_{j}\right)$, a cell cannot be dyed twice. Also, $t_{i}=0$ indicates that the cell is never dyed $c_{i}$ and $t_{j}=0$ indicates that it is never dyed $c_{j}$, but with respect to $l\left(p_{i}, p_{j}\right)$, the cell must be dyed either $c_{i}$ or $c_{j}$. This constraint for singular primary chromatic codes means that the cells are unable to dye both $c_{i}$ and $c_{j}$, or neither $c_{i}$ nor $c_{j}$.

Equation (23) is not the only constraint subjected to singular codes. Complete constraints can be transformed to an equivalent of partitioning an $n$-order complete graph G. In G, a vertex represents an entity and an edge represents the dyed-boundary induced by the two adjacent vertices of the edge. The $\mathrm{G}$ needs to be partitioned into $n$ subgraphs $\mathrm{G}_{1}$, $\mathrm{G}_{2}, \ldots, \mathrm{G}_{n}$, where $\mathrm{G}_{i}$ consists of a vertex $p_{i}$ and $S\left(\lambda_{i}\right)$ edges. Figure 7 is an example to partition a 4-order complete graph. Note, for some types of ACD, the above constraints might be invalid. For example, in half ACD (hACD) (see Section 5), it is possible to find a cell with code $\{0,0,3,3\}$.

Chromatic codes could also be employed to analyze point pattern. Given a cluster $\xi\left(\zeta_{1}, \zeta_{2}, \ldots, \zeta_{k}\right)$ and an entity $p_{j}$, the sum of $p_{j}$ 's saturations in $\xi$ is called $p_{j}$ 's local influence in the cluster and is denoted by $\boldsymbol{I}_{\mathrm{L}}\left(\xi, p_{j}\right)$, that is,

$$
\boldsymbol{I}_{\mathrm{L}}\left(\xi, p_{j}\right)=\sum_{i=1}^{k} \boldsymbol{I}\left(\zeta_{i}[j]\right), \zeta_{i} \in \xi
$$

and $p_{j}$ 's global influence $\boldsymbol{I}_{\mathrm{G}}\left(p_{j}\right)$ is defined from all cells, that is,

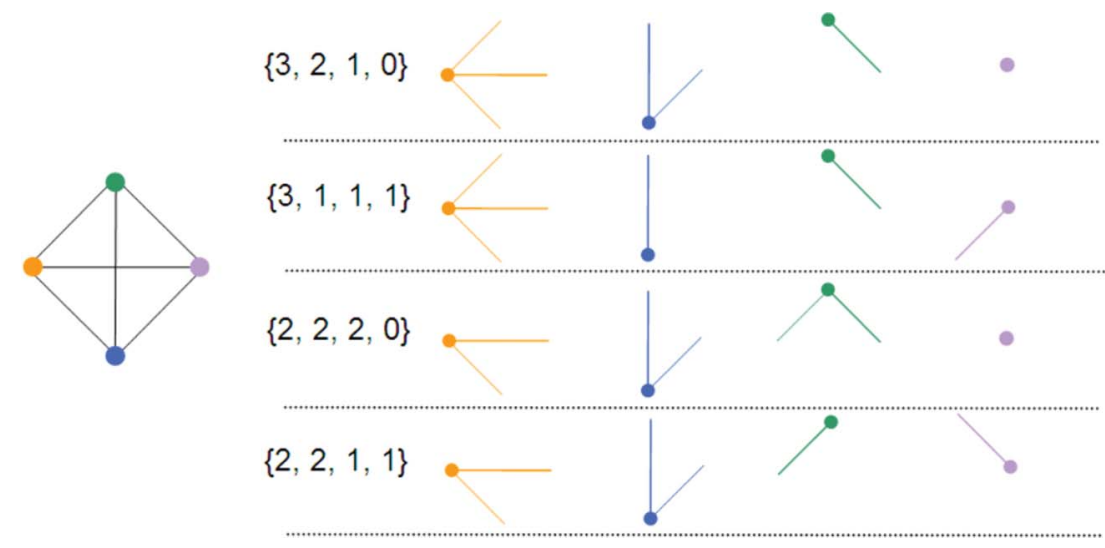

Figure 7. Graph partition corresponding to the primary chromatic codes of four entities. 

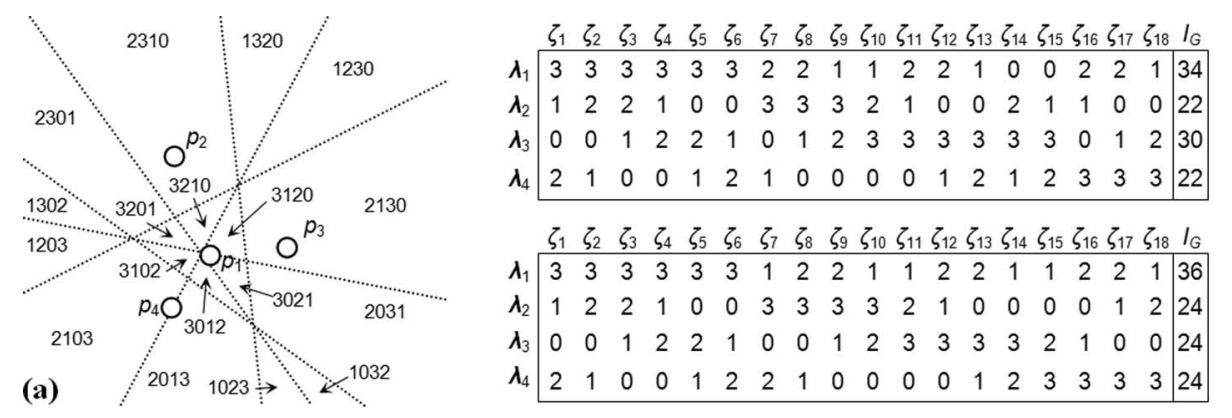

Figure 8. (a) A point pattern that an entity $p_{1}$ is in the center. (b) Global influences $\left(\boldsymbol{I}_{\mathrm{G}}\right)$ of entities with the pattern in Figure 2. (c) Entities' $\boldsymbol{I}_{\mathrm{G}}$ with the pattern in (a).

$$
\boldsymbol{I}_{\mathrm{G}}\left(p_{j}\right)=\sum_{i=1}^{D(n)} \boldsymbol{I}\left(\zeta_{i}[j]\right), \zeta_{i} \in \mathcal{A}(P)
$$

Given the four entities $\left\{p_{1}, p_{2}, p_{3}, p_{4}\right\}$ with distribution of Figure 2, the global influence of each entity, namely, the sum of all numbers in each row, is listed in the last column of Figure $8 \mathrm{~b}$. The entity $p_{1}$ has the most global influence 34 , and $p_{2}$ and $p_{4}$ both have the least global influences 22 . When their pattern is similar to that in Figure 8a, where $p_{1}$ locates at the center of the space, their global influences turn to $\boldsymbol{I}_{\mathrm{G}}\left(p_{1}\right)=36, \boldsymbol{I}_{\mathrm{G}}\left(p_{2}\right)=\boldsymbol{I}_{\mathrm{G}}\left(p_{3}\right)=\boldsymbol{I}_{\mathrm{G}}\left(p_{4}\right)=24$ (see Figure $8 \mathrm{c}$ ). These results indicate that for a given entity in space, the closer to the center it is, the more global influence it bears. Assuming points in set $\boldsymbol{P}$ distribute evenly in the space, $\boldsymbol{P}$ generates an ACD where cells also distribute homogeneously. In such an ACD, when an entity locates at space center, it occupies more cells as its nearest neighbors (where its saturation is $n-1$ ) hence with more global influence, but if an entity locates at space margin, it occupies more cells as its $k$ th-nearest neighbors (where its saturation is $n-k$ ) hence with less global influence. It is similar to put a speaker in a square center and more people could hear it. If we put it at the corner, then more people are margined and hence cannot hear the sound clearly.

We noticed that the whole space would be represented by a cluster merged from all cells, that is, $\boldsymbol{A}(\boldsymbol{P})=\xi\left(\boldsymbol{I}_{\mathrm{G}}\left(p_{1}\right)\right.$, $\left.\boldsymbol{I}_{\mathrm{G}}\left(p_{2}\right), \ldots, \boldsymbol{I}_{\mathrm{G}}\left(p_{n}\right)\right)$. For example, the space occupied by four entities such as in Figure 2a could be coded by $\xi(34,22,30$, $22)$, whereas those in Figure 8 a could be coded by $\xi(36,24$, $24,24)$. From the two examples, we can see that if two point sets have the different spatial patterns, they will generate different chromatic codes accordingly. Inversely, if we do not know the pattern of a given point set, we can calculate the chromatic code of the whole space and then derive its point pattern.

\section{Summary and future work}

A new tessellation model, SCT, was proposed for spaceentity modeling. Based on the half-plane partition and arrangement of lines, $\mathrm{ACD}$, especially the $\mathrm{OACD}$, was studied as an example of SCT. The basic concepts, operations, applications as well as other important aspects of SCT and ACD were introduced. In SCT, cells are assigned characteristic chromatic codes. These codes bear useful information that could be applied to analyze the relationships of space-to-space, entity-to-entity, and entity-to-space. Furthermore, these structured codes are flexible and feasible for geographical statistics and computation. The generation and the application of SCT follow three principles: partitioning, dyeing, and merging. In the real world, many systems coincide with the similar principle. For example, wild animals mark their habitats with their scent, cells with genetic information aggregate to tissues and organs, and atoms with internal particles combine to molecules and substance.

Compared with raster model and Voronoi diagrams, SCT is a spatial tessellation tightly connecting space and entities a more general perspective to represent space-entity relationship. We can take chromatic codes as a kind of objectorientated coordinates of the space. Recalling Euclidean coordinates $(x, y)$ in raster model or a text label $(\mathrm{a}, \mathrm{b}, \mathrm{c}, \mathrm{d})$ in Voronoi diagrams, we can see that they are too specific on depicting either space or entity attributes. A chromatic code like $(3,2,0,1)$ combines the merits of raster and Voronoi - it can be applied not only to spatial computation, similar to using Euclidean coordinates $(x, y)$, but also to entity interpretation, similar to using Voronoi label (a, b, c, d).

SCT is a quite novel model and holds many implications waiting for more explorations. Some mathematical tools will be helpful for this model, such as the theory of graph, permutation group, and combinatorics. In this article, we are unable to cover everything of SCT. Here, we just briefly discuss some related issues and future works.

\subsection{Complex chromatic space}

We would like to remind the readers that ACD is only one type of SCT. In practice, the generation and application of SCT could be generalized as a three-step scheme: partitioning space, dyeing entity attributes, and merging cells. How 
to implement the scheme is fairly flexible, depending on what the real entity is, what the real space is, and what the problem we want to solve is. Here we only studied OACD, the simplest form of ACD. In OACD, entities are points and dyed-boundaries are bisectors. The real world, however, is much more complicated than OACD. Geographical entities could be linear and areal objects and space are three dimensional. The dyed-boundary is also a broad concept in SCT. For shape, a dyed-boundary could be a curve, a zigzag, or even a zone; for objectivity, it could be real, virtual, or even fuzzy; and for location, it could be not only in the middle of two entities but also anywhere in the given space. Each spatial partition is always with respect to two entities. The essence of partitioning is that two entities use something, namely the dyed-boundary, to distinguish its own subspace from the others. As the next step, they need to mark their subspace. This is the issue of how to dye space. In OACD, entity index is the only attribute dyed into space, but real entities often have more additional attributes, either qualitative or quantitative. Any attribute is able to dye the space. The essence of dyeing is that entities give their own attributes to each subspace, so that the subspace turns to be cell inheriting those attributes. These cellular attributes need to be organized further, just like we arrange the cellular color $(1,1,1,2,2,4)$ into a chromatic code $(3,2,0,1)$. When cellular attributes are organized to chromatic codes, they are all converted into a few numbers for future computations.

Thus, after partitioning and dyeing, we transform the space from a blank into an organism. At this stage, we could even remove all entities. The coded and discretized space can independently exist. To further reveal the feature of the new space, the spatial chromatic statistics is an important approach.

\subsection{Spatial chromatic statistics}

In ACD, the rule is a type of chromatic statistics that is performed by chromatic codes. The cellular attributes, or chromatic codes, could be organized as a full-color table that is convenient to store in database. However, unlike the traditional database table, the spatial chromatic statistic is computable not only on columns but also on rows. The two types of statistics correspond to two perspectives from which we view the full-color table: entity-based or cellbased. The entity-based statistics could be used in the analysis of how space influences an individual entity, and the cell-based could be used in the analysis of how entities influence the space.

When more entity attributes are dyed into cells, they make more tables similar to the full-color table. All these tables provide additional information for spatial statistics and analysis. Moreover, the full-color table is not the only source for spatial chromatic statistics. By merging, cells disappear whereas clusters arise. Consequently, we can organize the chromatic codes of clusters into a new table. The subsequent merging, if necessary, could be based on the statistic variables of the new table. This way is a hierarchical merging from cells to small clusters then to large clusters.

\subsection{Algorithm, data structure, and implementation}

Due to the partitioning manner, the algorithm of OACD is simple. We just need to iteratively (1) compute and store the vertices that are generated by every current dyed-boundary intersecting all the previous, (2) split all involved previous cells into new cells, and (3) re-dye all cells (update their new saturations in full-color table). Consequently, ACD provides a new algorithm to generate various Voronoi diagrams. For example, the $k$ th-nearest point Voronoi regions are easily generated by executing a simple SQL query:

SELECT*FROM FullColourTable WHERE $t[i]=n-k+1$

Then the retrieved cells can be merged and hence form the Voronoi region of the $i$ th entity. Repeating the above query $n$ times (let $i=1$ to $n$ ), we obtain the $k$ th-nearest point Voronoi diagrams that are generated by the $n$ entities.

In a traditional relational database, one row corresponds to a record and one column corresponds to an attribute. To store a full-color table in a relational database, we may take either cells or entities as the records in rows. However, because the number of cells is usually greater than that of the entities, it is better to store cells as records. On the other side, the statistic queries in traditional relational database are often based on the attributes, that is, the columns of a table, and therefore for full-color tables in the database, it is inconvenient to deal with all kinds of spatial merging, because they are based not only on cell-based but also sometimes on entity-based rules. Therefore, we suggest that in future the full-color table should be better treated as a matrix rather than a database table.

\subsection{Chromatic codes, spatial relations, and index}

Mathematically, chromatic codes are the arrangement of entity indices. The relationship between entities and space is well established in these codes that are analogous to genetic codes. Transition numbers could be thought as a kind of topological chromatic distance. When two clusters have close colors, they also have close topology. In spatial index, chromatic codes could be used as a type of index. Traditional spatial index uses minimum bounding rectangle (MBR) or convex hulls. In ACD, we may use minimum bounding clusters because spatial relations are easy to compute through chromatic codes.

In ACD, chromatic codes represent cellular and cluster's spatial relations simply and explicitly. Our main concern, however, is the spatial relations between entities, especially for lines and polygons. One possible approach 
is first abstracting any entity as a point, partitioning and dyeing the space, and then using this chromatic space to dye entities backward. Then we can reason their spatial relations based on their new colors. In Section 2, we have demonstrated spatial relations between cells and cells, 2-cell and 2cell clusters, 3-cell and 3-cell clusters, but we still need to figure out the general spatial relations between any $n$-cell and $m$-cell clusters.

\subsection{Half arranged chromaticity}

Given an entity set $\boldsymbol{P}$, if every pair of entities induces a dyedboundary, then the induced ACD is called a full ACD (fACD). According to Equations (1) and (2), $n$ entities will induce $L(n)=\mathbf{O}\left(n^{2}\right)$ dyed-boundaries and subsequently $D(n)=\mathbf{O}\left(n^{4}\right)$ cells, so for a very large $n$, the number of dyed-boundaries, cells, and color vectors per cell will be too large to be handled in GIS. However, in our geographical world, when two entities are so far apart from each other, they usually have no relationship. Consequently, for such a pair of entities, it is unnecessary to induce their dyedboundary and dye the space, and hence a fACD turns to the so-called hACD. For example, we can set a real distant threshold, say, $1000 \mathrm{~m}$, to determine whether we need to induce a dyed-boundary. Or more realistically, we could combine hACD to a small-world network. In this case, if two people or entities are socially connected, they may induce a dyed-boundary. The number of dyed-boundaries and cells in hACDs could be sharply reduced to $\mathbf{O}(n)$ and $\mathbf{O}\left(n^{2}\right)$, respectively. From the perspective of spatial resolution, the number of cells in an ACD is similar to the number of pixels in a raster image. If we define the number of cells as the resolution of an ACD, then the resolution of the fACD is the highest $\mathbf{O}\left(n^{4}\right)$. The resolution of hACD is much lower than a fACD's. If we do not connect any pair of entities, then the resolution is 1 (there is no spatial tessellation). Therefore, ACD's resolutions range from 1 to $\mathbf{O}\left(n^{4}\right)$. Comparatively, in a traditional raster model, the range of pixel amount can be technically from 1 to infinite if a pixel size is set as small as possible.

hACD is more realistic and applicable than fACD. We developed a computer program to generate $\mathrm{ACD}, \mathrm{NACD}$, and hACD; see Figure 9. In hACD, the cellular primary chromatic codes have more variations. For example, in full

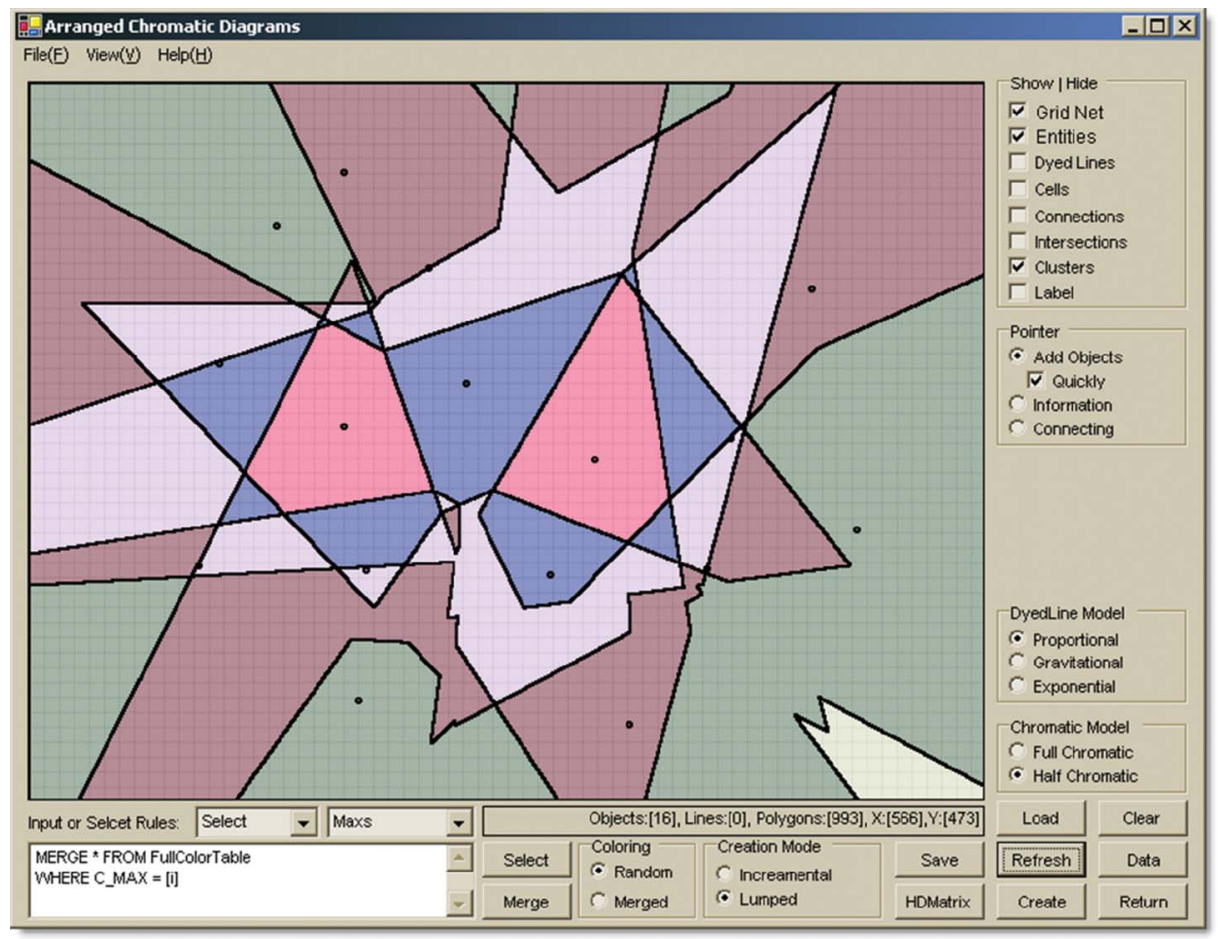

Figure 9. Graphical user interface of ACD program and an example of hACD generated by 16 entities. If the distance between the two entities is greater than 200 pixels, then they are assumed to have no relationship and hence we do not generate their dyed-boundary. Cells were merged in Rule 5, which is implemented by 'MERGE*FROM FullColorTable WHERE C_MAX $=[i]$.' Rule 5 is slightly different from Rule 1 that forms ordinary Voronoi diagrams. This diagram is an NACD where the subregions with the same color represent a cluster. C_MAX is a statistical variable indicating the maximal saturation of a chromatic code. In a full OACD, C_MAX is always $n-1$, but in a half OACD, C MAX of some chromatic codes will be smaller. For this example, assuming the 16 entities are shops, we could observe two central regions, the red polygons (cluster) in this figure, where C_MAX is 6, showing that some shops have the greatest local impacts within the red cells. This diagram implies that the geographical distance tends to form a space that contains a few local centers instead of only one global center. 
OACD, primary chromatic code is always $\{0,1,2, \ldots, n\}$, but in hACD, a cell is probably with color $\{0,0,2,3, \ldots, n\}$, where the first two zeros indicate that for each dyedboundary like $l\left(p_{1}, p_{i}\right)$ or $l\left(p_{2}, p_{i}\right)$, the cell is always dyed the color $p_{i}$. In addition, because entities $p_{1}$ and $p_{2}$ have no relationship, they do not induce $l\left(p_{1}, p_{2}\right)$, so both $p_{1}$ and $p_{2}$ 's colors have not been dyed into the cell at all. Therefore, if given a cell with chromatic code segment ' 0,0 ' like in $\{0,0$, $2,3, \ldots, n\}$, then we will know not only that the distance between $p_{1}$ and $p_{2}$ is quite far but also that the cell is far away from both $p_{1}$ and $p_{2}$. We also found many interesting hACD features that are very different from the Voronoi diagrams. For instance, different entities share the same nearest neighborhood.

\section{Acknowledgments}

This work is supported by the Faculty Research Grant No. 21644 provided by the University of Massachusetts, Amherst.

\section{References}

Aurenhammer, F., 1991. Voronoi diagrams - a survey of a fundamental geometric data structure. ACM Computing Surveys, 23, 345-405.

Berchtold, S., Bohm, C., and Kriegel, H.-P., 1998. The pyramidtree: breaking the curse of dimensionality. In: Proceedings ACM SIGMOD international conference on management of data. Seattle, WA: ACM Press, 142-153.

Chen, J., et al., 2001. A Voronoi-based 9-intersection model for spatial relations. International Journal of Geographical Information Science, 15, 201-220.

Cohn, A.G., 1992. A hierarchical representation of qualitative shape based on connection and convexity. In: A. U. Frank and W. Kuhn (ed.), Spatial information theory: a theoretical basis for GIS,Vol. 988: Lecture notes in computer science. Berlin: Springer-Verlag, 311-326.

Dramstad, W.E., Olson, J.D., and Forman, R.T.T., 1996. Landscape ecology principles in landscape architecture and land-use planning. Washington, DC: Harvard Graduate School of Design, Island Press, American Society of Landscape Architects.

Egenhofer, M.J. and Franzosa, R.D., 1991. Point-set topological spatial relations. International Journal of Geographical Information Science, 5, 161-176.

Egenhofer, M.J. and Herring, J., 1991. Categorizing binary topological relationships between regions, lines, and points in geographic databases. Orono, ME: Department of Surveying Engineering, University of Maine.

Faloutsos, C. and Roseman, S., 1989. Fractals for secondary key retrieval. College Park, MD: University of Maryland.
Frank, A.U., 1996. Qualitative spatial reasoning: cardinal directions as an example. International Journal of Geographical Information Science, 10, 269-290.

Gold, C.M., 1994. Review: spatial tessellation - concepts and applications of Voronoi diagrams. International Journal of Geographical Information Science, 8, 237-238.

Goodchild, M.F., 1992. Geographic data modelling. Computers and Geosciences, 18, 401-408.

Guttman, A., 1984. R-trees: a dynamic index structure for spatial searching. In: Proceedings of the1984 ACM SIGMOD international conference on management of data. Boston, MA: ACM Press, 47-57.

Klein, R., 1989. Concrete and abstract Voronoi diagrams. In: R. Klein (ed.), Lecture notes in computer science. New York: Springer-Verlag, Vol. 400.

Kovalevsky, V.A., 1989. Finite topology as applied to image analysis. Computer Vision, Graphics, and Image Processing, 46, 141-161.

Laurini, R. and Thompson, D., 1992. Fundamentals of spatial information systems. New York: Academic Press.

Lehmann, F. and Cohn, A.G., 1994. The EGG/YOLK reliability hierarchy: semantic data integration using sorts with prototypes. In: Proceedings of the $3 r d$ international conference on information knowledge management. New York: ACM Press, 272-279.

Lo, C.P. and Yeung, A.K.W., 2002. Concepts and techniques of geographic information systems. Upper Saddle River, NJ: Prentice Hall.

Longley, P.A., et al., 2005. Geographic information systems and science. Chichester: John Wiley \& Sons.

Okabe, A., Boots, B., and Sugihara, K., 1992. Spatial tessellations: concepts and applications of Voronoi diagrams. Chichester: John Wiley \& Sons.

O'Rourke, J., 1998. Computational geometry in C. Cambridge: Cambridge University Press.

Papadias, D. and Theodorodis, Y., 1997. Spatial relations, minimum bounding rectangles, and spatial data structures. International Journal of Geographical Information Science, $11,111-138$.

Randell, D.A., Cui, Z., and Cohn, A.G., 1992. A spatial logic based on regions and connection. In: Proceedings of the $3 r d$ international conference on principles of knowledge representation and reasoning. San Francisco, CA: Morgan Kaufmann Publisher, 165-176.

Roy, A.J. and Stell, J.G., 2001. Spatial relations between indeterminate regions. International Journal of Approximate Reasoning, 27, 205-234.

Schneider, M., 1999. Uncertainty management for spatial data in databases: fuzzy spatial data types. In: Advances in spatial databases, the 6th international symposium, SSD'99,Vol. 1651: Lecture notes in computer science. Berlin: SpringerVerlag, 330-351.

Winter, S., 1995. Topological relations between discrete regions. In: M. J. Egenhofer and J. R. Herring (ed.), Advances in spatial databases, Vol. 951: Lecture notes in computer science. Berlin: Springer-Verlag, 310-327. 


\section{Appendix}

This appendix gives a proof of the first property of OACD, that is, there are no equicolor cells in OACD. To prove it, let us start from two lemmas.

Lemma 1: In an $O A C D$ created by $\boldsymbol{P}=\left\{p_{1}, p_{2}, \ldots, p_{n}\right\}$, cellular chromatic codes are always the permutations of $\{0,1,2, \ldots$, $n-1\}$.

Proof: According to the definition of a dyed-boundary of OACD, any point $p$ that is equidistant from any two entities $p_{i}$ and $p_{j}$, that is, $d\left(p, p_{i}\right)=d\left(p, p_{j}\right)$, only locates on dyed-boundaries. Therefore, in the plane $\Re^{2}$, point $p$ inside any cell is not equidistant from all entities. Then for a given cell, $\boldsymbol{P}$ can be rearranged to $\left\{p_{1}{ }^{\prime}, p_{2}{ }^{\prime}, \ldots\right.$, $\left.p_{n}^{\prime}\right\}$ with the below order:

$$
d\left(p, p_{1}^{\prime}\right)<d\left(p, p_{2}^{\prime}\right)<\cdots<d\left(p, p_{n}^{\prime}\right)
$$

This order indicates that for $n-1$ dyed-boundaries $l_{1}\left(p_{1}{ }^{\prime}, p_{2}{ }^{\prime}\right)$, $l_{2}\left(p_{1}{ }^{\prime}, p_{3}{ }^{\prime}\right), \ldots, l_{n-1}\left(p_{1}{ }^{\prime}, p_{n}{ }^{\prime}\right)$, the cell is dyed with $p_{1}{ }^{\prime} n-1$ times, for $n-2$ dyed-boundaries $l_{1}\left(p_{2}{ }^{\prime}, p_{3}{ }^{\prime}\right), l_{2}\left(p_{2}{ }^{\prime}, p_{4}{ }^{\prime}\right), \ldots, l_{n-2}\left(p_{2}{ }^{\prime}, p_{n}{ }^{\prime}\right)$, the cell is dyed with $p_{2}{ }^{\prime} n-2$ times, and so on. Therefore their cellular chromatic codes are always the permutations of $\{0,1,2, \ldots, n-1\}$.

For the permutations of $\{0,1,2, \ldots, n-1\}$, an E-transposition $(a, b)$, denoted by $T e(a, b)$, is an exchange of only two elements $a$ and $b$ but with all others staying the same. For example, $T e(1,2)$ turns code $(3,2,1,0)$ to $(3,1,2,0)$. There is another type of transposition $(a, b)$, L-transposition, denoted by $T l(a, b)$. LTransposition only exchanges two elements whose locations (indices) are $a$ and $b$, regardless of what they are. For example, $\operatorname{Tl}(1,2)$ turns code $(3,2,1,0)$ to $(2,3,1,0)$. Here, we can see that the result of an action $\operatorname{Te}(a, b)$ may not equal to that of $\operatorname{Tl}(a, b)$. An E-transposition could be converted to an equivalent Ltransposition if their results are same. For example, $T e(1,2)=T l$ $(2,3)$, they both turn $(3,2,1,0)$ to $(3,1,2,0)$.

Let us consider two scenarios: (1) Two cells $\zeta_{1}$ and $\zeta_{2}$ are adjacent with a dyed-boundary $l(i, j)$; $(2)$ acting $T l(i, j)$ on $\zeta_{1}$. Actually scenario (2) turns $\zeta_{1}$ to $\zeta_{2}$ and hence makes the scenario (1). We call this turn moving a step from $\zeta_{1}$ to $\zeta_{2}$ by passing $l(i, j)$. If $T l(i, j)=T e(a, b)$, it is also called $\zeta_{1}$ 's transition from $a$ to $b$, and the absolute difference between $a$ and $b,|a-b|$, is called the $E$ transposition number of the transition.

Lemma 2: In an OACD, moving a step from $\zeta_{1}$ to $\zeta_{2}$ by passing a dyed-boundary $l(i, j)$ is equivalent to acting $T l(i, j)$ on $\zeta_{1}$ with $E$ transposition number 1.

Proof: The generation of two adjacent cells $\zeta_{1}$ and $\zeta_{2}$ is equivalent to using $l(i, j)$ to partition an initial polygon. Assume $l(i, j)$ is the last dyed-boundary partitioning the polygon. Before the partition, the polygon is expressed as

$$
\left(\begin{array}{c}
\ldots, i, \ldots, j, \ldots \\
\ldots, x, \ldots, y, \ldots
\end{array}\right)
$$

where the bottom row represents the chromatic code of the polygon and the top row represents code locations or indices. The code indices are always fixed from 1 to $n$. Before the partition of $l(i, j)$, the $i$ th saturation is $x$ and the $j$ th is $y$. The ellipses indicate that at these locations saturations have been already decided by previous partitions and they keep unchanged for the action $\operatorname{Tl}(i, j)$.

Let $x$ and $y$ have a relationship $y=x+k$, and $k \geq 0$, then the polygon could be rewritten as

$$
\left(\begin{array}{c}
\ldots, i, \ldots, j, \ldots \\
\ldots, x, \ldots, x+k, \ldots
\end{array}\right)
$$

After the partition of $l(i, j)$, the polygon is decomposed to two new cells and they are dyed with color $c_{i}$ and $c_{j}$, respectively. Then their chromatic codes could be written, respectively, as

$$
\zeta_{1}\left(\begin{array}{c}
\ldots, i, \ldots, j, \ldots \\
\ldots, x+1, \ldots, x+k, \ldots
\end{array}\right)
$$

and

$$
\zeta_{2}\left(\begin{array}{c}
\ldots, i, \ldots, j, \ldots \\
\ldots, x, \ldots, x+k+1, \ldots
\end{array}\right)
$$

According to Lemma 1, the codes of $\zeta_{1}$ and $\zeta_{2}$ should be both the permutations of $\{0,1, \ldots, n\}$. If $k=1$, then in $\zeta_{1}$, two saturations are both $x+1$. This contradicts Lemma 1 . If $k>1$, then in $\zeta_{1}$, there must be a saturation $x$ hidden in its ellipses and so as in $\zeta_{2}$ 's. Therefore in $\zeta_{2}$, two saturations will be equal to $x$. This also contradicts Lemma 1 . As a result, $k$ can only be 0 . That implies $x=y$, and therefore their chromatic codes could be rewritten, respectively, as

$$
\zeta_{1}\left(\begin{array}{c}
\ldots, i, \ldots, j, \ldots \\
\ldots, x+1, \ldots, x, \ldots
\end{array}\right)
$$

and

$$
\zeta_{2}\left(\begin{array}{c}
\ldots, i, \ldots, j, \ldots \\
\ldots, x, \ldots, x+1, \ldots
\end{array}\right)
$$

Checking their locations and corresponding saturations, we can see that elements at locations $i$ and $j$ are exchanged and the difference between the two elements is 1 .

Corollary 1: In OACD, if two cells share an edge then their transition number is 2.

Proof: According to Equations (A6) and (A7) in the proof of Lemma 2, their transition number is $|(x+1)-x|+\mid x-(x+1)$ $\mid=2$.

The steps that a cell walks to its adjacent cells one by one form a path. Given a cell $\zeta$ with an edge $l(i, j)$, the other cells that locate in the half-plane $\boldsymbol{H}(j, i)$ are called $\zeta$ 's half-outside cells with respect to $l(i, j)$ (Figure A1). Therefore, in an OACD, with respect to $\zeta$ 's all edges, any cell is $\zeta$ 's half-outside cell except $\zeta$ itself.

Theorem 1: If $\xi$ is the set of $\zeta$ 's half-outside cells with respect to $l$ $(i, j)$, then for any $\zeta_{\text {out }} \in \xi, \zeta \neq \zeta_{\text {out }}$.

Proof: Let us represent a chromatic code using a graph (Figure A2). In Figure $\mathrm{A} 2 \mathrm{a}$, each vertex represents a location $V$ and also an element with saturation $S$, and each edge represents a possible Ltransposition and also an equivalent E-transposition. According to Lemma 2, moving a step is equivalent to swapping two vertices with E-transposition number 1. A cell walking within the half-plane $\boldsymbol{H}(j$, $i$ implies that it passes the dyed-boundary $l(i, j)$ only once, that is, $T l$ $(i, j)$ is no longer allowed, so in the graph, we erased the edge between $V_{i}$ and $V_{j}$. By the action of $T l(i, j), S_{m}$ and $S_{m+1}$ are swapped to $V_{j}$ and $V_{i}$ (Figure A2b). In order to prove this theorem, we actually 


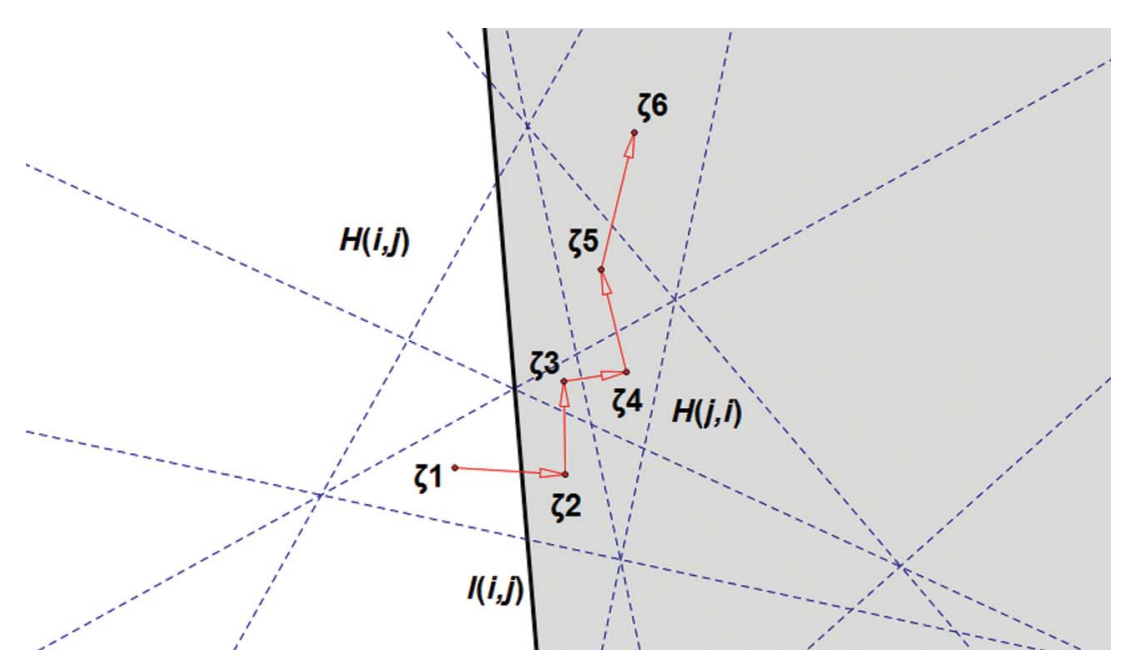

Figure A1. With respect to $l(i, j), \zeta_{1}$ moves a step to $\zeta_{2}$, and then subsequently four steps to $\zeta_{6}$. These five steps make a path from $\zeta_{1}$ to $\zeta_{6}$. The shadow cells are $\zeta_{1}$ 's half-outside cells with respect to $l(i, j)$. They comprise the half-plane $\boldsymbol{H}(j, i)$.
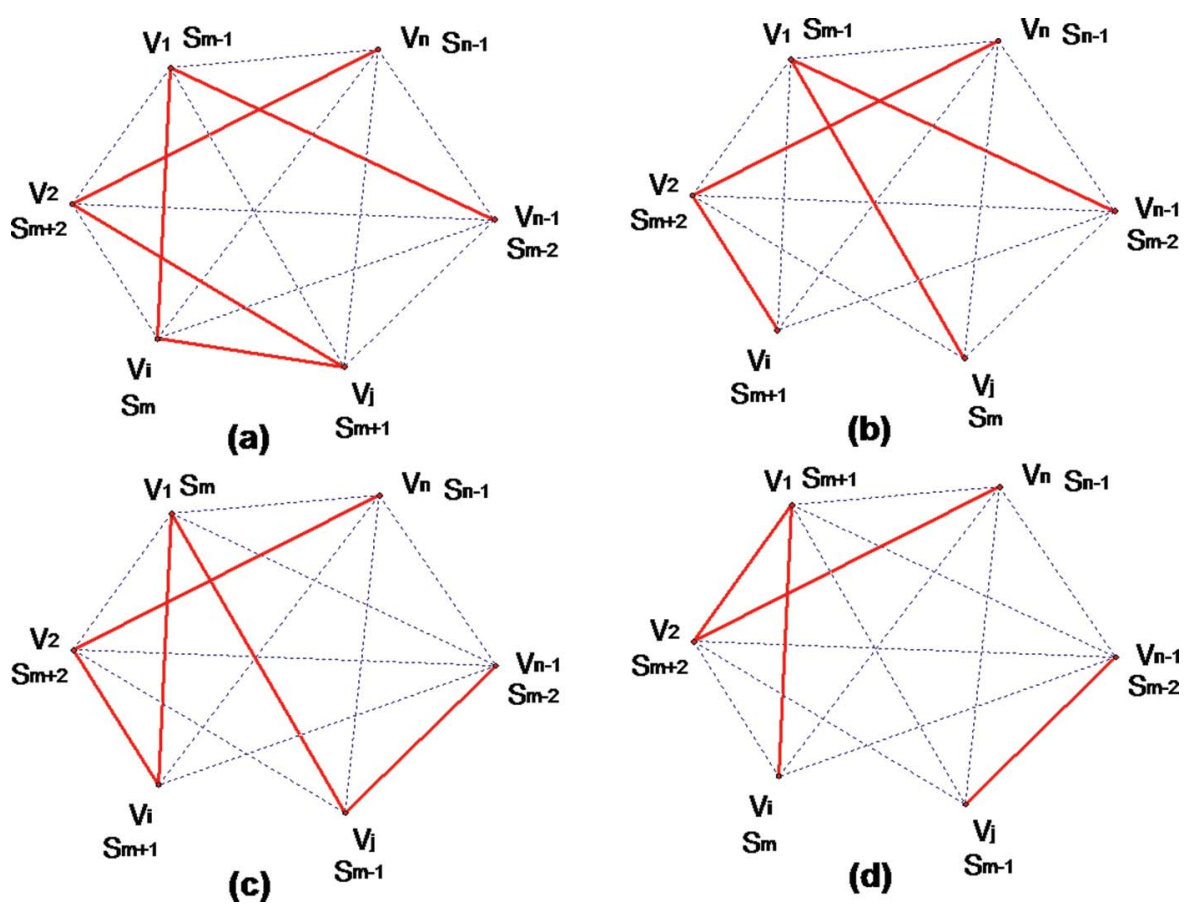

Figure A2. The four graphs demonstrate chromatic codes and moving steps. Vertices are code locations and always fixed from 1 to $n$, whereas saturations are swapped between two vertices with E-transposition number 1. The solid lines represent the legal swaps. Moving from (a) to (b) is an action of $\operatorname{Tl}\left(V_{i}, V_{j}\right)=T e\left(S_{m}, S_{m+1}\right)$. Then the edge $\left(V_{i}, V_{j}\right)$ is the cutoff. Moving from (b) to (c) is an action of $T e\left(S_{m-1}, S_{m}\right)$, and (c) to (d) is $\operatorname{Te}\left(S_{m}, S_{m+1}\right)$. In the case (d), $S_{m+1}$ is impossible to return $V_{j}$ again.

need to prove that no matter where the cell walks to, two elements $S_{m}$ and $S_{m+1}$ are impossible to return their original respective locations $V_{i}$ and $V_{j}$, if given the following two constraints:
(1) A vertex swap is only allowed (legal) if its E-transposition number $=1$.

(2) The vertex swap between $V_{i}$ and $V_{j}$ is not allowed. 
If we first act $T e\left(S_{m-1}, S_{m}\right)$ (from Figure A2b to c) and next $T e\left(S_{m}\right.$, $S_{m+1}$ ) (from Figure A2c to d), then $S_{m}$ returns $V_{i}$. At this time, we fix $S_{m}$ and try to swap $S_{m+1}$ to $V_{j}$. Because $V_{i}$ and $V_{j}$ are disconnected, at $V_{j}, S_{m-1}$ is only allowed to swap with $S_{m-2}$, and $S_{m-2}$ is only allowed to swap with $S_{m-3}$, and so on. Because at $V_{j}$, the saturation becomes smaller and smaller, $S_{m+1}$ is impossible to be swapped to $V_{j}$. Similar scenarios will occur if we first move $S_{m+1}$ to $V_{j}$ : the saturation at $V_{i}$ will become larger and larger. The above two scenarios indicate that it is impossible to let $S_{m}$ and $S_{m+1}$ return to their initial locations simultaneously. Consequently, at least at either $V_{i}$ or $V_{j}, \zeta$ 's saturation is not equal to that of its half-outside cells, namely, $\zeta$ and $\zeta_{\text {out }}$ are not equicolor cells. $\square$

Corollary 2: There are no equicolor cells in an $O A C D$.

Proof: Given a cell $\zeta$ whose edges consist of a series of dyedboundaries, according to Theorem $1, \zeta$ is not equal to all corresponding half-outside cells with respect to one dyed-boundary. Because any cell can be regarded as $\zeta$ 's half-outside cell with respect to a specific dyed-boundary, therefore all other cells are not equal to $\zeta$. 